\title{
Optimal insurance portfolios risk-adjusted performance through dynamic stochastic programming
}

\author{
Giorgio Consigli Vittorio Moriggia Sebastiano Vitali Lorenzo Mercuri
}

\begin{abstract}
The practical adoption of the Solvency II regulatory framework in 2016, together with increasing property and casualty (PC) claims in recent years and an overall reduction of treasury yields across more developed financial markets have profoundly affected traditional risk management approaches by insurance institutions. The adoption of firm-wide risk capital methodologies to monitor the companies' overall risk exposure has further consolidated the introduction of risk-adjusted performance measures to guide the management medium and long-term strategies. Relying on a dynamic stochastic programming formulation of a 10 year asset-liability management (ALM) problem of a PC company, we analyse in this article the implications on capital allocation and risk-return trade-offs of an optimization problem developed for a global insurance company based on a pair of risk-adjusted return functions. The analysis is relevant for any institutional investor seeking a high risk-adjusted performance as for regulators in their structuring of stress-tests and effective regulatory frameworks. The introduction of the concept of risk capital, or economic capital, in the definition of medium and long term insurance strategies poses a set of modeling and methodological issues tackled in this article. Of particular interest is the study of optimal ALM policies under different assets' correlation assumptions. From a computational viewpoint it turns out that, depending on the assumed correlation matrix, the stochastic program is linear or of second order conic type. A case study from a real-world company development is presented to highlight the effectiveness of applied stochastic programming in capturing complex risk and return dynamics arising in modern corporate finance and lead to an efficient long-term financial allocation process.
\end{abstract}

Keywords Property and casualty liabilities · Dynamic stochastic programming · Risk capital allocation - Return on risk-adjusted capital $\cdot$ Surplus investment return

\section{Introduction}

In this article we present the key features of an asset-liability management (ALM) problem formulated as a dynamic stochastic program (DSP) [see Bertocchi et al. (2011) as general reference and for a specific ALM model] for a property and casualty (PC) insurance company.

At an enterprise-wide level, we assume the PC company as an agent, whose economic performance depends on three divisions: the technical division, which is liability-oriented, concentrating the core PC business with claims and operational costs; the investment division, which is fundamentally asset-oriented, where the investment strategy is defined; and the risk management division, whose aim is to assess and monitor over time the risk exposure generated by the technical and investment businesses. Throughout this article the investment manager of a PC portfolio is regarded 
as the relevant decision maker: she/he is assumed to reflect the decisions of a strategic investment committee, whose aim is to determine the company's strategic asset allocation (SAA).

Record PC insurance claims reported by global players in recent years (see CEA 2016) and the final adoption in 2016 of the Solvency II regulatory framework (following European Parliament 2009) have induced a majority of global PC firms to increase their technical reserves and at the same time revise their capital allocation policies under growing market competition. An integrated approach to risk and asset-liability management (ALM) by insurance companies has emerged at the beginning of this decade as a managerial requirement and resulted in several internal modeling efforts, see Mulvey et al. (2007) and Consigli et al. (2011).

The debate on PC companies' regulatory capital requirements dates back to the early 90s when the Actuarial Advisory Committee to the NAIC PC Risk-Based Capital Working Group issued a study putting down the key principles to be considered in future legislative efforts (see NAIC P/C RBC working group and others 1992): core to the study was the establishment of a direct relationship between a PC firm's capital and its exposure to risk, from which the concept of risk based capital (RC). From the UK glossary of actuarial terms the RC is defined as the theoretical amount of capital needed to absorb the risks of operating a business with financial obligations to customers and market agents. This is the amount necessary to ensure that the business has an acceptably low probability of becoming financially insolvent: as such it is also referred to as Capital at Risk (CaR), whose statistical equivalent is the Value-at-Risk (see Jorion 1996). Following that approach and a sequence of partial EU regulatory directives, Solvency II provided in 2016 a comprehensive framework 
introducing the concepts of Solvency Capital requirements (SCR) which taking an extremely conservative approach was associated with a RC shortage carrying a $0.5 \%$ probability of occurrence over a 1 year period. In this article we consider the above (internal) RC estimate as the reference concept for SCR assessment and focus on two main aggregate sources of risk, respectively associated with technical and investment portfolios. Once a SCR-consistent RC estimate is generated, the PC management is faced with a capital allocation problem of the resulting amount across the business units so to reach the company strategic objectives. This is referred to as a $\mathrm{RC}$ allocation problem, commonly faced by any financial or insurance intermediary. In principle the capital allocation phase thus implies the definition of a strategy and in this respect we distinguish between the two. We go more in detail in these concepts in Sect. 3 .

Since the early 90's we have thus witnessed a significant set of contributions addressing from different perspectives the key topic of an optimal risk capital allocation on insurance firms' profitability. Relevant early contributions from different methodological and modellng perspectives are Denault (2001), Gaivoronski et al. (2001), Dhaene et al. (2003), Mulvey et al. (2007), Tasche (2007), Buch and Dorfleitner (2008), Saita (2010), Alessandri and Drehmann (2010) and Maume-Deschamps et al. (2015).

Contributions in this area can be roughly clustered in three groups: those focusing on capital allocation from the perspective of optimal capital structures and with an economic and regulatory perspective, which date back to the early stages and to NAIC P/C RBC working group and others (1992); those addressing the risk capital allocation problem from the perspective of risk measures theory which originated from Artzner et al. (1999) on risk measures axiomatics and, finally, those relying on ALM models and stochastic programming (SP) approaches as in Cariño et al. (1994), Dempster et al. (2003), Gaivoronski et al. (2001), Mulvey and Erkan (2003) and Consigli et al. (2011). This article relies on recent developments in this latter area and by integrating in a realistic set-up the risk-capital philosophy behind the first group of works, leads to new modeling and computational challenges in the area of quantitative institutional ALM, see Consigli and Dempster (1998), Mulvey and Erkan (2003) and Bertocchi et al. (2011).

After the seminal paper by Cariño et al. (1994) focusing, as here and in Consigli et al. (2011), on a real-world application of SP techniques, a rich set of contributions originated from the extended cooperation with the insurance sector by Mulvey, who concentrates in Mulvey and Erkan (2005) and Mulvey et al. (2007) on the general structure of the decision process for multinational insurers operating in global markets and the implications on the optimal capital allocation decisions. The general relationship between risk measures and capital allocation was considered in Dhaene et al. (2003), while in de Lange et al. (2004) a stochastic programming approach was adopted to model a PC reinsurance problem. The interplay between capital allocation and the return on risk-adjusted capital has been analysed in a one period setting by Buch et al. (2011).

We follow up from those contributions and focus on the relationship between risk capital constraints and risk-adjusted performance of a PC portfolio in a multistage framework and derive a relevant set of evidences for risk management and regulatory purposes. The ALM model introduced in Sect. 2 is consistent with modern accounting 
standards and employs a terminology to a certain extent industry-specific, whose economic rationale we try to clarify throughout.

This research work highlights the effectiveness of a dynamic SP formulation and its suitability to address a complex insurance management problem. We extend previous contributions in this area through (i) the explicit introduction in a dynamic model of control equations on the risk capital evolution (as a function of the portfolio strategy),

(ii) the evaluation of the impact of different correlation assumptions on the investment $\mathrm{RC}$ and the problem solution, and (iii) the definition of an optimal RC allocation consistent with two risk-adjusted return targets. The article is primarily motivated by a modeling challenge but, through the development, leads to interesting computational evidences: specifically the derivation of a stochastic linear program associated with a statistical assumption of perfectly positively correlated asset returns, typical of a market crisis.

By focusing on 1-year risk estimates and associated capital allocations, furthermore, quantitative ALM approaches were until recently unable to incorporate strategic objectives over long horizons, despite this being both an internal company and a regulatory objectives. The extension of the planning horizon to 10 years is shown in the case study to lead to positive risk-adjusted returns under stable RCresources.

The article is structured as follows: in Sect. 2 we introduce the key elements of a modern approach to risk- and asset-liability management of an insurance PG portfolio and in 2.2 the ALM model mathematical instance. In the following Sect. 3 we focus on the features of the recently introduced risk-based capital framework, whose modeling and mathematical implications are considered in Sects. 3.1 and 3.2 respectively. The impact of alternative correlation assumptions on the optimal strategy are also considered relying on the concept of risk-adjusted returns. In Sect. 4 we first summarize different solution approaches and then provide an extended set of numerical evidences for model validation and scenario analysis focusing on a PC portfolio problem.

\section{Insurance riskand asset-liability management}

We extend the PC ALM model in Consigli et al. (2011) to include explicitly a pair of risk-adjusted performance measures and a company's risk capital dynamic equation, formulated in accordance with the main Solvency II requirements. The ALM model develops from the definition of core actuarial variables, related to revenues and costs of the PC technical division, to consider portfolio strategies generating a given investment performance. We focus specifically on two risk-adjusted performance measures [see Modigliani and Modigliani (1997) for an introduction]: the so-called investment value surplus (IVS) and the popular return on risk-adjusted capital (RoRAC). By introducing this pair of risk-adjusted return targets in the ALM model, we link the definition of the optimal strategy to a sufficient return generation and an effective control of the risk exposure. 


\subsection{Modeling approach}

Let $t \Phi 0,0.5,1,2,3,5,10$ be the problem's planning horizon: we consider a 10 year problem in which the portfolio manager seeks a SAA driven by two return targets. She/he manages an investment portfolio which over time will benefit from cash surpluses generated by the core PC activity and must comply with the RC constraints produced by the risk management division. Independently the technical business also faces a risk capital constraint.

The 10 year horizon allows the inclusion of the IVS and RoRAC targets at the 3and 10-year horizons, respectively. The ALM model is formulated according to a multistage stochastic programming approach leading to an optimal decision tree process and an underlying discrete representation of the uncertainty. The non-homogeneous increasing time lengths of the stages from half year to 5 years at the last stage reflects the strategic nature of a strategic long-term ALM problem.

\subsubsection{General notation}

We adopt a canonical scenario tree representation of the model of uncertainty (see Consigli et al. 2012) with nodal labels $n \in N_{t}$ over $t \in T . N_{T}$ defines the set of leaf nodes at the terminal horizon or number of scenarios, sample paths from the root to the leaf nodes. We indicate with $t_{n}$ the time stage of node $n$ and denote the set of immediately descending, or children nodes of $n$ by $n$ and its unique parent node by $n$-: for each leaf node $n N_{T}$ the sequence of nodes $n, n-\ldots, n_{\sigma}$ defines a scenario nodal sequence where $n_{0}$ is the root node. Then, we denote with $\omega_{n}$ a scenario is a sample path from the root to a leaf node $n$. We will use $\omega \notin \Omega, F, P)$ to denote a random tree process defined in a probability space with sample space $\Omega, \sigma$-algebra $F$ and probability measure P. Furthermore, we denote by $a(n)$ the sequence of all ancestors for any node $n$. The set of nodes originating from node $n$ up to the end of the planning horizon will instead be denoted by $c(n)$ : this is the subtree originating from $n$. The model objective function includes two stages with associated targets: we denote with $t^{j}$ the timing of target $j$ so that ${ }_{\ell} N_{t}$ will be the corresponding nodes in the tree. Under this convention, the conditional expectation over the nodesen $N_{t}$ with respect to information available in $t$ is denoted by $\mathrm{E} \cdot \|\left[F_{t}\right]$, for $\boldsymbol{\epsilon} T$. All scenarios are assumed equally probable.

Under this convention every random process is a discrete non-recombining tree process and we enforce non-anticipativity implicitly (see Dupačová et al. 2000) with every control action by the decision maker taken under uncertainty: the first at the root node under full uncertainty, while the (last) decision at the beginning of the last period still facing a residual uncertainty associated with the last possible data realizations.

\subsubsection{Decision variables}

We consider a SAA problem with 5 asset classes and 7 investment opportunities plus cash. The investment universe is denoted by $A:=A_{1} \cup A_{2} \cup A_{3} \cup A_{4} \cup A_{5}$ with asset classes $A_{j}: j=1$ corresponds to Treasury benchmarks, $j=2$ to corporates, $j=3$ to equity, $j=4$ to alternative investments and $j=5$ to real 
estate. Treasuries and corporates, include two investment options each: plain vanilla (Barcaps, see below) or inflation-linked government (Tips) benchmarks the former and investment or speculative (high yield) grade the latter asset class. The PC portfolio manager represents the reference decision entity in this problem: the following decision variables are considered.

$\begin{array}{lll}x_{i, m, n} & \text { amount of asset } i \text { held in node } n \text { and bought in node } m & \in a(n) \\ x_{i, n}^{+} & \text {amount of asset } i \text { bought in node } n \\ x_{i, m, n}^{-} & \text {amount of asset } i \text { sold in node } n \text { and bought in node } m \in a(n) \\ z_{n} & \text { cash surplus in node } n\end{array}$

By aggregating the above we also denote: $\overline{X_{\bar{r}, n}} \quad x_{i}$ the investment in asset $i$ in node $n$, thus $X_{n}=i_{A} X_{i, n}$ for the overall portfolio value in node $\underline{n}$. Aggregate new investments and selling ${ }^{n}$ doe isions in node $n$ are denoted by $X_{n}^{+} \quad i A x_{i, n}^{+}$and $X_{n}^{-} \quad i \overline{\bar{A}} \quad m a(n) x_{i, m, n}^{-}$, respectively. At time $0, x_{i, n 0}=$ is the current asset allocation, before the solution of the SP problem. Slightly abusing notation we indicate with $X^{+}, X^{-}$the decision vectors in the objective function (4) to reflect the overall buying and selling decisions over the planning horizon.

\subsubsection{PC model variables and parameters}

The ALM model instance relies on an extended set of model variables, that we present next distinguished by division. The adopted notation follows Consigli et al. (2011) and canonical insurance terminology.

1. Technical division:

$R_{n} \quad$ amount of PC premiums collected in node $n$

$L_{n} \quad$ insurance claims paid in node $n$

$C_{n}$ operational costs paid in node $n$

$\Pi_{n}^{t} \quad$ cumulative technical profit in node $n$

$\bigwedge_{n, t}$ liability reserves for claims expiring in $t$

$\Lambda_{n}$ liability reserves in node $n$. Are computed relying on a Chain ladder methodology as $\Lambda_{n}=t>t_{n} \Lambda_{n, t}$, i.e. as the sum of liability reserves expiring in any $t$ after $t_{n}$ until the claims extinction years, see Consigli et al. (2011) and Weindorfer (2012)

$\Delta_{n}^{\wedge}$ duration of the liabilities in node $n$. It is computed as $\Delta_{n}^{\wedge}={ }^{-}{ }_{t>t_{n}} \wedge_{n, t}(t-$ $\left.t_{n}\right)$. Both $\Lambda_{n}$ and $\Delta^{\wedge}$ are assumed to be estimated exogenously and input to the model

2. Investment division:

$\Delta_{n}^{X}$ duration of the asset portfolio in node $n$. It is given by $\Delta^{X}={ }_{i}^{-} \quad-$ $x_{i, m, n} \Delta_{i, n}$ where $\Delta_{i, n}$ is the duration of asset $i$ in node $n$.

$\Pi_{n}^{f}$ cumulative portfolio trading profit in node $n$

$U_{n} \quad$ potential (in case of selling) portfolio gain and losses in node $n$

$\zeta \quad$ internal funding cost rate. It is given by $\zeta=\max \left\{1 \%, r_{z, n_{0}}\right.$ where $r_{z, n_{0}}$ is the return on the cash account in the root node 
$\Phi_{n} \quad$ internal cumulative funding costs in node $n$ faced by the portfolio manager. It is computed as $\Phi_{n}=\Lambda_{n^{-}} \zeta\left(t_{n}-t_{n^{-}}\right)+\Phi_{n^{-}}$

3. Risk management division:

$K_{n}^{a}$ actuarial or technical risk capital (TRC) in node $n$. It represents the 1year CaR (99.5\%) of the division. In this model the TRC is assumed to be a constant proportion of the company reserves and denoted $b_{n}=K^{a} \mathbf{K}_{\mathbb{x}}^{a} \Lambda_{n}$, where the coefficient $\mathrm{K}^{a}$ is estimated by the actuarial division

$K_{n}^{f_{1}}$ interest rate risk exposure in node $n$ due to asset-liability (A-L) duration mismatching. Also referred to as ALM risk capital.

$K_{n}^{f_{2}}$ market risk capital in node $n$.

$K_{n}^{f}$ investment risk capital (IRC) in node $n$. It represents the $\mathrm{VaR}(99.5 \%)$ over the $t_{n}-t_{n-}$ period. It is computed as $K^{f} \overline{\bar{n}} K^{f 1}{ }_{n} K^{f 2} \cdot{ }_{n}$

$\hat{K}^{-}{ }_{n}^{f}$ maximum tolerable investment risk in node $n$.

$K_{n} \quad$ total risk capital in node $n$ faced by the company over the period $t_{n}-t_{0}$. It is given by $K_{n}=K_{n}^{a} K_{n}{ }^{f}$ At an enterprise-wide level this is also referred to as Economic capital.

\subsubsection{Other parameters}

The following random factors will determine the optimal investment policy, as clarified in Sect. 2.2.

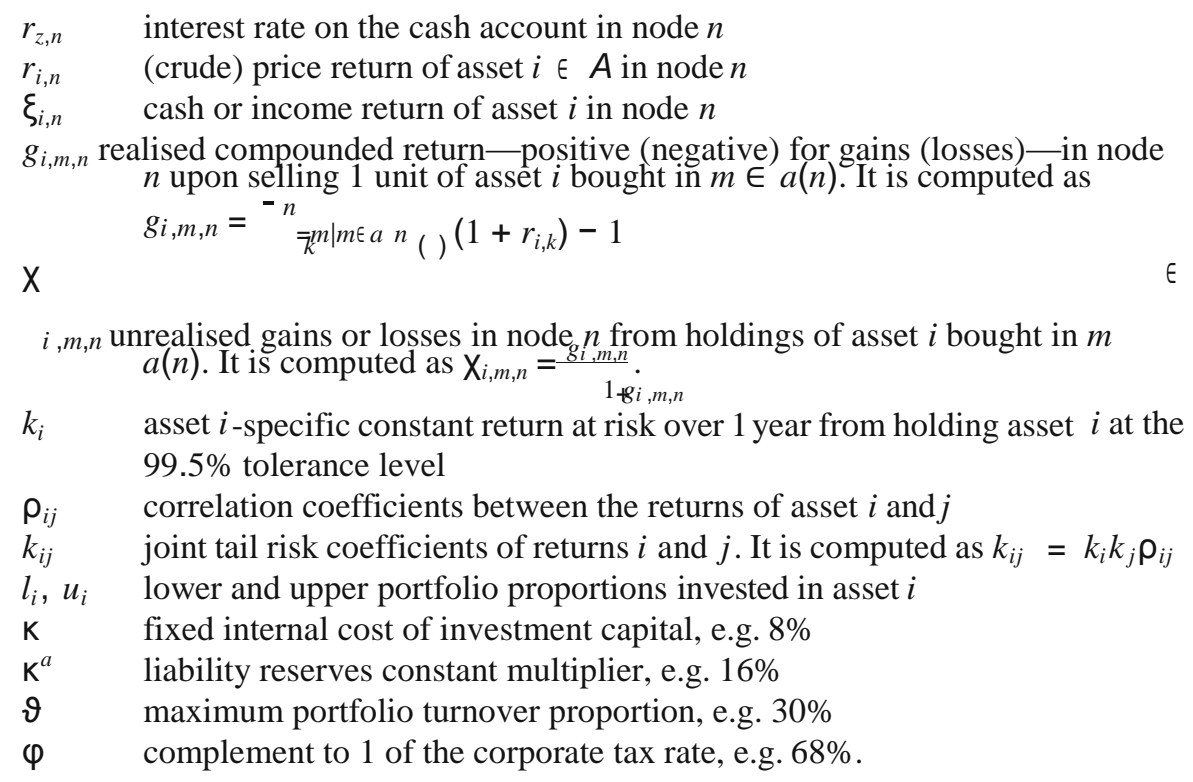

\subsubsection{Objective function}

The objective function in the proposed SAA problem, relies on the definition of the investment value surplus and the return on risk-adjusted capital. The IVS belongs to the 
class of so-called economic value added (EVA) performance measures (see Saita 2010) while the RoRAC is an enterprise-wide, time-independent, corporate risk-adjusted return ratio. Their definition in this model is consistent with corporate requirements and in this setting is motivated by the distinction between actuarial- and investmentbased profits and RC variables. When pursuing an optimal investment plan, the PC manager will target two risk-adjusted return goals associated with the IVS and the RoRAC.

The IVS is defined by

$$
\mathrm{v}_{n}^{1}=\left(\Pi^{f}{ }_{n} \Phi_{n}\right) \varphi-\mathrm{K} K^{f}, \quad n \in N_{t}, t \in T
$$

This is a portfolio reward measure, determined, net of corporate taxes $\varphi$, by subtracting from the portfolio trading profit $\Pi_{n}^{f}$ first an internal cost of funding $\Phi_{n}$ and then the cost of the IRC: $\mathrm{\kappa} K_{n}^{f}$. All quantities in this IVS definition are set to 0 at $t=0$. Furthermore let

$$
\mathrm{v}_{n}^{2}=\Pi^{f}{ }_{n^{+}} U_{n}+\Pi^{a}, \quad n \in N_{t}, t \in T
$$

be the company cumulative profit in node $n$ defined by the sum of the investment and technical profits: unlike for the IVS, we consider in the investment profit both the investment trading profit $\Pi_{n}^{f}$ and the unrealised gains and losses $U_{n}$. The RoRAC process is

$$
\Psi_{n}=\frac{\bigvee^{2}}{K_{n}^{\prime}} \quad n \in N_{t}, t \in T
$$

in terms of compounded returns per unit risk exposure.

The management is assumed to set a 3-year IVS target $\tilde{V}^{1}$ and a 10-year RoRAC target $\tilde{\Psi}$ : we denote with $\tilde{\mathrm{V}}_{n}^{2}=\tilde{\Psi} K_{n}$ the associated company target profit. The portfolio manager is assumed to minimize the sum of the expected shortfalls, when positive, with respect to the targets at the $t^{1}=3$ and $t^{2}=10$ year horizons:

$$
\min _{X^{+}, X^{-}}\left\{\lambda \mathbb{E}\left[\left(\tilde{v}^{1}-v_{n}^{1}\right)^{+} \mid \mathscr{F}_{t^{1}}\right]+(1-\lambda) \mathbb{E}\left[\left(\tilde{v}_{n}^{2}-v_{n}^{2}\right)^{+} \mid \mathscr{F}_{t^{2}}\right]\right\}
$$

where $\lambda \in(0,1)$.

Ceteris paribus, the portfolio manager will always opt for the strategy requiring the least capital allocation. The economic motivation behind the adoption of these two risk-adjusted returns in an Institutional ALM problem relates first to the market practice by major financial institutions to announce publicly their short and medium term RoRAC targets and then, specific to this model instance, to the adoption of an internal competitive scheme in which the technical and investment divisions compete to achieve a given target, and the need to decompose the company profit among them arises. In what follows we focus specifically on the investment division. 


\subsection{ALM model instance}

The optimization problem is formulated as a targets' shortfall minimization problem under an extended set of constraints, to be satisfied almost surely (a.s.):

$$
\min _{X^{+}, X^{-}}\left\{\lambda \mathbb{E}\left[\left(\tilde{v}^{1}-v_{n}^{1}\right)^{+} \mid \mathscr{F}_{t^{1}}\right]+(1-\lambda) \mathbb{E}\left[\left(\tilde{v}_{n}^{2}-v_{n}^{2}\right)^{+} \mid \mathscr{F}_{t^{2}}\right]\right\}
$$

subject, for all $n \in N_{t}, t \in T$, to the following constraints. We set $\Pi_{n=}^{a}=\Pi_{n 0} \stackrel{f}{=} 0$ and

$$
\begin{aligned}
x_{i, n_{0}-, n_{0}}= & x_{i, n_{0}-}+x_{i, n 0}^{+}-x_{i, n_{0}-, n_{0}}^{-} \forall i \\
x_{i, m, n}= & \sum_{m \in a(n)} x_{i, m, n-}\left(1+r_{i, n}\right)+x_{i, n}^{+}-\sum_{m \in a(n)} x_{i, m, n}^{-} \forall i \\
l_{i} X_{n} \leq & X_{i, n} \leq u_{i} X_{n} \forall i \\
X_{n}^{+}+X_{n}^{-}= & \vartheta \sum_{i} X_{i, n-}\left(1+r_{i, n}\right) \\
z_{n}= & \sum_{i} \sum_{m \in a(n)} x_{i, m, n}^{-}-\sum_{i} x_{i, n}^{+}+R_{n}-L_{n}-C_{n} \\
& +\sum_{m \in a(n-)} x_{i, m, n-} \xi_{i, n}+z_{n-}\left(1+r_{z, n}\right) \\
\Pi_{n}^{a}= & R_{n}-L_{n}-C_{n}+\Pi_{n-}^{a} \\
\Pi_{n}^{f}= & \sum_{i} X_{i, n-} \xi_{i, n}+\sum_{i} \sum_{m \in a(n)} x_{i, m, n}^{-} g_{i, m, n}+\Pi_{n-}^{f} \\
U_{n}= & \sum_{i} \sum_{m \in a(n)} x_{i, m, n} \chi_{i, m, n} \\
v_{n}^{1}= & \left(\Pi_{n}^{f}-\Phi_{n}\right) \phi-\kappa K_{n}^{f} \\
v_{n}^{2}= & \Pi_{n}^{f}+U_{n}+\Pi_{n}^{a} \\
K_{n}^{f_{1}}= & \left(\Delta_{n-}^{X}-\Delta_{n-}^{\Lambda}\right) \Delta r_{z, n}\left(t_{n}-t_{n-}\right)+K_{n-}^{f_{1}} \\
K_{n}^{f_{2}}= & \sum_{\sum_{i \in \mathscr{A}} \sum_{j \in \mathscr{A}} X_{i, n-} X_{j, n-} k_{i j}\left(t_{n}-t_{n-}\right)+K_{n-}^{f_{2}} .} . \\
K_{n}^{f}= & K_{n}^{f_{1}}+K_{n}^{f_{2}} \\
K_{n}^{f} \leq & X_{n}-\Lambda_{n}^{f}-\Delta K_{n-}^{a}+K_{n-}^{f} \\
K_{n}^{a}= & \kappa^{a} \Lambda_{n} \\
K_{n}= & K_{n}^{f}+K_{n}^{a} \\
& \\
&
\end{aligned}
$$


The solution of the SP problem (4) under the constraints (5)-(21) will generate an optimal strategy $X^{o}$, for $n N_{t}, t T$.

The set of inventory balance constraints (5) and (6) determines the portfolio evolution along the tree with a final possible revision at the beginning of the last stage. At the root node we assume an initial input portfolio $x_{i, n 0^{-}}$which is revised through sellings $x_{i, n_{0}-, n_{0}}^{-}$and buyings $x_{i, n_{0}}^{+}$to determine the optimal here-and-now implementable decision $x_{i, n 0}$.

Equations (7) and (8) define lower and upper bounds on individual portfolio positions and turnover constraints proportional to the nodal portfolio value: $l_{i}$ and $u_{i}$ are policy bounds aimed at avoiding portfolio concentrations and induce a diversification which is judged appropriate by the portfolio manager, while $\vartheta$ in (8) will limit the overall buying and selling decisions in node $n$ to a given proportion of the current portfolio value.

The cash balance $z_{n}$ evolves as indicated in (9) due to selling and buying decisions, premiums inflows $R_{n}$ net of casualty $L_{n}$ and operational costs $C_{n}$, and the revenues ${ }_{m}$ $a_{a(n)} x_{i, m, n} \xi_{i, n}$ generated in node $n$ from holdings in the ancestor node $\overline{\bar{m}}$, plus compounded interests in the cash account.

Equations (5) to (9) are standard in the ALM literature Bertocchi et al. (2011) with the only noticeable difference that here we keep track of the holding periods $t_{n} t_{m}$ between nodes $n$ and $m \in a(n)$ : such modeling choice is motivated by the need to account for stage specific realised and unrealised investment profits. The constraints that follow from (10) to (21) are instead problem specific.

The set of Eqs. (10), (11) for $\Pi_{n}^{a}$ and ${ }_{n}$ allows the derivation of the cumulative nodal technical and investment profit as determined by actuarial revenues and costs and by income and capital gains on selling decisions. Equation (12) quantifies the sum of realised and potential investment profits.

The objective function and the constraints from (13) to (19) incorporate the main modeling contribution of this article. In a one period setting the definition of an optimal capital allocation with respect to the RoRAC was considered by Buch et al. (2011) and Stoughton and Zechner (2007). Mulvey et al. (2007) laid down the key elements for deriving a dynamic model of the risk capital in a PC context. Gaivoronski et al. (2001) had previously considered the implications of a regulatory capital constraint on a SP formulation of a PC company problem within the Norwegian market. From a mathematical viewpoint, again within a more general SP formulation Pflug and Roemisch (2007) laid down the foundations of risk measurement and control in a dynamic framework and Pflug and Ruszczynski (2005) focused on dynamic risk measures properties. The novelty here, even if under relatively simple statistical assumptions, is on the inclusion of risk-adjusted returns and risk capital equations in a multistage model, where portfolio rebalancing in node $n \mathbb{N}_{t}$ for $t<T$ will jointly determine the returns and the risk capital protection from the current stage to the children nodes $n_{+}$. The investment portfolio risk exposure in Eq. (17) is decomposed in a component related to the asset-liability duration gap in Eq. (15) and one associated with market risk in Eq. (16): both quantities affect the IVS and the RoRAC evolution. The first quantity will depend on the duration gap and the increments of the money market rate: $\Delta r_{z, n}$. 
In Eq. (18) for $n \in N_{t}$, finally we require the investment risk capital increment not to exceed the current portfolio value net of liabilities and the technical risk capital: as explained in Sect. 3.2 this is regarded internally by the management as a safety measure. This constraint is relevant to the risk manager and employs in this setting a structural approach to default risk estimation [see Consigli et al. (2012), Das and Tufano (1996) and Crouhy et al. (2000) on credit risk models and associated risk control problems]: from Eq. (18), we derive in the case study a specific scenario $\hat{\bar{K}}_{n}^{f}$ to be regarded as a default boundary beyond which the PC portfolio would no longer be sufficient to cover potential losses.

The solution of the ALM problem will primarily depend on the input asset and liability scenarios, on the targets and, crucially, on the statistical assumptions underlying the investment risk estimation. We consider in particular three instances of the correlation matrix $\rho_{i j}^{h}, h=1,2,3$ to define $k_{i j}^{h}=k_{i} k_{j} \rho^{h}{ }_{i j}$ in Eq. (16):

$-\rho^{1} \mp\left\{I_{i j} ;\right\}$ for uncorrelated asset returns, in which different markets behave independently one another and portfolio diversification is expected to be highly beneficial to control investment risk.

- $\rho^{2} \beta^{2}\{$ is a jij conservative Solvency II-compliant correlation matrix, typically taken from an internal risk model validated by the regulatory body (see Consigli et al. 2011).

$-\rho^{3} \oplus\left\{1_{i j}\right\}$ fer perfect positive asset correlations featuring a market crisis condition, thus leading to a worst-case scenario in terms of market risk.

Accordingly, we formulate and solve an SP instance for every correlation matrix. Each SP problem solution will determine an optimal investment strategy or contingency plan $X_{\rho^{h}}^{o}=\left\{X_{n, \rho}^{o}\right\}$ for $n \in N_{t}, t \in T$ from the root node to the end of the decision horizon, a corresponding RC evolution $K_{\rho^{h}}^{f}=\left\{K_{n, \rho^{h}}^{t}\right\}$, a maximum investment risk $\hat{\bar{K}}_{n, \rho^{h}}$, a resulting IVS $v^{1}=\left\{\mathrm{v}^{1}\left(X_{n}^{o}, K_{\rho^{h}}^{f}\right)\right\} \underset{\rho^{h}}{\text { and }}$ RoRAC $\Psi_{h}=\left\{\Psi_{n}\left(X^{o}, K^{f}\right)\right\}_{\rho^{h}}$ We focus on the three correlation matrices' implications in Sect. 3.

\section{Riskmanagement of a PC portfolio}

Following the Solvency II agreement [see European Parliament (2009) and subsequent directives, which led to the final 2016 practical adoption], every year the management of an Insurance company is informed by the risk manager of the current SCR together with an RC estimate for the forthcoming year: we assume here that in its budget planning the management will then determine a set of profit targets and allocate the $\mathrm{RC}$ among business units to achieve those targets. This is the risk budgeting or RC allocation problem which is emerging as a standard in the banking and insurance industry.

We assume in this article that the core PC technical division will determine the required RC protection as a given constant proportion of the evolving PC reserves. Furthermore, as far as the investment division is concerned, two main risk sources are considered: one associated with interest rates and one with asset values market dynamics. The former will affect the IRC according to the prevailing A-L duration 
gap. The latter will affect the IRC through the risk coefficients $k_{i}, \ldots i A$ and the adopted correlation matrix $\rho^{h}, h \exists, 2,3$. Following from the previous section, a correlation matrix $\rho^{2}$ is assumed to be validated by the regulatory body and consistent with Solvency II. In this setting the optimal strategy $X_{\rho^{2}}^{o}$ is taken as Solvency compliant and the RC evolution consistent with external solvency requirements. We distinguish two phases: before the solution of the optimization problem, the RC estimate can be interpreted as the capital needed to hedge any future potential loss, an output of the risk management division. After the solution of the problem, in view of the introduced targets, we can refer to $K_{\rho^{2}}^{f}$ as the RC absorbed by the investment policy or to be allocated by the management across business units. The introduction of the upper bound $\hat{\bar{K}}_{\rho^{h}}$ in Eq. (19) can then be interpreted as the maximum possible scenario-dependent investment risk exposure which is a-priori sustainable by the current portfolio. A long-term strategy aims at preserving the maximum possible distance from such upper bound and at the same time minimize the risk to go below the Solvency II requirements: the management on the other hand has an interest to minimize the (expensive) cost of capital at an aggregate level.

In a multiperiod model, the interaction between optimal investment policy and risk capital becomes central to the analysis (see Buch and Dorfleitner 2008; Dhaene et al. 2003; Stoughton and Zechner 2007). We show in the case study in Sect. 3.2 that the optimal portfolio strategy generated by the SP solution, under different correlation assumptions will lead to a perfectly hedged interest risk exposure $K^{f_{1}}$ and a minimal $K^{f_{2}}$. From a computational viewpoint as the assumption on assets' correlation changes, the resulting stochastic program will be either linear (SLP) or second-order conic (SOCP) leading to an interesting comparative analysis.

\subsection{Risk capital allocation over 1 year}

Consider a simple (yet standard in the industry) 1-year problem and assume a RoRAC target $\tilde{\Psi}$ and a RC estimation based on the regulatory matrix $\rho^{2}$. We have $K^{f}{ }_{n}=$ $K_{n}^{f 1}+K_{n}^{f 2}$, capturing the interest and the market risk exposure. In general fixed-income positions will be motivated by risk constraints and the need of portfolio immunization against interest rates random fluctuations. Let $\sqrt{6} N_{1}$ and assume a current, time 0 , investment portfolio $X_{n 0}$. The following steps are commonly adopted in practice:

1. The actuarial division will determine, for current expected liability cash flows and term structure of interest rates, the PC liability value at the root node $\Lambda_{n 0}$;

2. Given $\bigwedge_{n 0}$ and the current investment portfolio. $X_{n}$ the risk management division will determine the technical and investment risk exposures $K^{d}$ and $K^{n}$ : those estimates depend on expected casualties, the current asset-liability duration gap and the capital-at-risk, as estimated here through regulatory-compliant risk coefficients and correlation matrix.

3. Given those estimates the top management will set the RoRAC target: the actuarial and the investment divisions face very different risk-return profiles and the technical and market risk exposures may also be assumed independent one another. 
per unit risk capital.

5. The profit actually generated by the two divisions and business units therein, will then be taken into account to assess the targets and the risk capital distribution over the following year and so forth.

A 1-year risk evaluation and capital allocation is consistent with a myopic policy but cannot accommodate a strategic view, nor it can rely on a 3-year planning, as required by industry standards. Furthermore companies solvency conditions can hardly be evaluated over such short horizons. A strong incentive has thus emerged to adapt modeling efforts and quantitative ALM strategies to longer horizons (see Alessandri and Drehmann 2010; Buch and Dorfleitner 2008). When extending to longer horizons, notice however that year 1 is splited in two semesters, in this way allowing a more accurate short-term planning.

\subsection{Investment risk capital in a dynamic setting}

In a dynamic model, every portfolio rebalancing decision will induce a new risk exposure over the following period. The ALM model specification focuses on an overall assessment of the profit generated by an investment policy and the associated risk capital.

To minimize the cost of investment capital the portfolio manager at every stage will minimize $K_{n}^{f 1}$ and $K_{n}^{f 2}$ at the end of the current stage. When extending the analysis to longer horizons beyond 1 year, market factors' statistical assumptions are typically required to be updated. Here below, taking however a conservative approach, we stick to an assumption of constant correlations and risk coefficients. Our interest is indeed on the definition of an optimal capital allocation over a 10-year horizon under a strategic view and the development of a comparative analysis: any future regulatory-compliant correlation matrix will surely fall in the $\rho^{1}-\rho^{3}$ range, while the $k_{i}$ are assumed to reflect long term marginal tail risks. In practice, under a standard approach Solvency II correlation matrices are revised only occasionally. Through Eqs. (19) and (18) in the ALM model, furthermore, we impose a condition strongly affecting the investment policy in the long run.

In Eq. (18) the maximum investment risk allowed on any node is defined by the difference between the portfolio value net of reserves and the increment of the technical risk exposure. Under any scenario the value of the portfolio must thus be sufficient to compensate the PC portfolio risk exposure over the entering stage. As the liability reserves and the portfolio structure change so will such upper bound. In particular increasing casualties may lead to negative actuarial profits and affect the economic capital: under such scenario the investment strategy is expected to compensate those losses but within the given risk limit. A scenario dependent maximum risk exposure allows the quantification of the distance between that upper bound and the risk exposure generated by the investment strategy. In the case study in Sect. 4.4 we show that by requiring a tight control on the investment risk capital the optimal strategy will effectively hedge interest and market risk sources and in this way strongly limit the capital protection needed by the portfolio policy.

In the next section we estimate for given scenario set and optimal policy, the sen- 
sitivity of the market risk exposure to different correlation matrices: the analysis will quantify the costs induced by increasingly conservative correlation assumptions on the risk capital and, consequently on the risk-adjusted performance.

\subsubsection{Sensitivity of market risk to assets' correlations}

Consider the process $K_{\rho^{\prime}}^{f 2}(\omega)$ along a scenario $\omega$ induced by a policy $X^{o}(\omega)$ and a correlation assumption $\rho^{h}, h \neq, 2,3$ : this process is evaluated in each node, but the root, relying on the portfolio allocation in the ancestor node, on the risk coefficients $k_{i}$ and on correlation assumption $\rho^{h}$. We wish to estimate the sensitivity of the RC evolution to different correlation assumptions after the problem solution. We derive to this aim a set of relevant inequalities. Then for each $\omega$ :

$$
K_{\rho^{1}}^{f_{2}}(\omega)<K_{\rho^{2}}^{f_{2}}(\omega)<K_{\rho^{3}}^{f_{2}}(\omega) .
$$

Consider any policy $X^{o}(\omega)=X(\omega)$. To derive the inequalities (22), notice that for $\rho$ $\rho_{3}=1$ o diagonal elements $k^{2}$ and off-diagonals $k_{i} k_{j}$. Thus:

$$
\begin{aligned}
K_{n, \rho^{3}}^{f_{2}} & =\sqrt{\sum_{i \in \mathscr{A}} \sum_{j \in \mathscr{A}} X_{i, n-} X_{j, n-} k_{i j}}\left(t_{n}-t_{n-}\right)+K_{n-, \rho^{3}}^{f_{2}} \\
& =\sqrt{\left(\sum_{i \in \mathscr{A}} X_{i, n-} k_{i}\right)^{2}\left(t_{n}-t_{n-}\right)+K_{n-, \rho^{3}}^{f_{2}}} \\
& =\sum_{i \in \mathscr{A}} X_{i, n-} k_{i}\left(t_{n}-t_{n-}\right)+K_{n-, \rho^{3}}^{f_{2}}
\end{aligned}
$$

The expression for $K_{n-\rho^{3}}^{f 2}$ becomes linear and the optimal problem is in this case solved as a stochastic linear program.

Similarly, for $\rho=\rho_{1}=I$ :

$$
\begin{aligned}
K_{n, \rho^{1}}^{f_{2}} & =\sqrt{\sum_{i \in \mathscr{A}} \sum_{j \in \mathscr{A}} X_{i, n-} X_{j, n-} k_{i j}}\left(t_{n}-t_{n-}\right)+K_{n-, \rho^{1}}^{f_{2}} \\
& =\sqrt{\sum_{i \in \mathscr{A}}\left(X_{i, n-} k_{i}\right)^{2}}\left(t_{n}-t_{n-}\right)+K_{n-, \rho^{1}}^{f_{2}} \\
& =\sqrt{\sum_{i \in \mathscr{A}} X_{i, n-}^{2} k_{i}^{2}}\left(t_{n}-t_{n-}\right)+K_{n-, \rho^{1}}^{f_{2}}
\end{aligned}
$$


Now, by the triangular inequality, given the nonnegative risk coefficients $k_{i}$ we also have:

$$
\left(\sum_{i \in \mathscr{A}} X_{i, n-} k_{i}\right)^{2}>\sum_{i \in \mathscr{A}}\left(X_{i, n-}^{2} k_{i}^{2}\right)
$$

Thus, the inequalities (22) must hold along any scenario $\omega$. The differences $\left(K_{\rho^{2}}^{f 2}-\right.$ $\left.K_{\rho^{1}}^{f 2}\right)$ and $\left(K_{\rho^{3}}^{f 2}-K_{\beta_{2}}^{f 2}\right)$ define scenario-wise the incremental capital resources requested by the strategy $X^{o}(\omega)$ because of a correlation change from $\rho^{1}$ to $\rho^{2}$ and to $\rho^{3}$, respectively.

\subsubsection{Risk manager and regulatory views}

Taking all scenarios into account and the associated optimal strategies, for $t \in T$, $n \in N_{t}$ we can derive $X_{\beta}^{o}$ and $K_{\rho^{h}}^{f}$ and generate two processes for $h=1,2$ :

$$
\begin{aligned}
& \mathrm{Y}_{2,1}^{f}=K_{n}^{f 2}\left(X^{o}\right)_{\rho^{2}}-K^{f 2}\left(X^{o}\right)_{\rho 1} \\
& \mathrm{Y}_{3,2}^{f}=K_{n}^{f 2}\left(X^{o} b^{3}-K^{f 2}\left(X^{o}\right)_{\rho^{2}}\right.
\end{aligned}
$$

In Eq. (25), $\mathrm{Y}_{2,1}^{f}$ and $\mathrm{Y}_{3,2}^{f}$ represent two processes which depend now on the optimal policies $X_{{ }^{h}}^{o}$ for $h=1,2,3$. Less relevant is in practice the case $\rho^{1}$ versus $\rho^{3}$.

The first difference quantifies the cost in terms of increased risk capital requirements of a correlation shift from $\rho^{1}$ to $\rho^{2}$. The second process quantifies instead the cost induced by a market crisis. Both quantities are of interest to regulators: the first process will define, ceteris paribus, the economic cost associated with the introduction of the regulatory framework, relative to a condition of uncorrelated market risks. The second process will provide information on the additional penalization induced by an extremely stressed market condition. A positive $\mathrm{Y}_{2,1}^{f}$ tree process will indicate a sure capital increase due to the correlation shift, while if along a specific scenario the process goes negative that would imply a RC saving induced by the associated investment policy.

Relying on the ALM problem solution, we can also define the following RC buffer tree process, which, following constraint (19), reflects instead the distance between the allocated risk capital and a maximum sustainable Capital at Risk:

$$
\delta_{n, \rho^{h}}^{f}(\omega)=\hat{K}_{n, \rho^{2}}^{f_{2}}(\tilde{\omega})-K_{n, \rho^{h}}^{f 2}(\omega)
$$


where $\hat{\bar{K}}_{n, \rho^{2}}^{f_{2}}(\tilde{\omega})$ is a user-defined upper bound associated with a specific scenario $\tilde{\omega}$ under correlation $\rho^{2}$. The risk manager is assumed to select that upper bound to determine a maximum tolerable loss by the portfolio manager.

A negative $\delta_{n, \rho^{h}}^{f}(\omega)$ along scenario $\omega$ would generate a theoretical default of the company. This process is of primary importance to the PC company risk manager and the portfolio manager since it provides, for given optimal strategy and market conditions, a measure of the risk capital that might be allocated before reaching a default condition.

\section{Computational study}

The stochastic program (4) under the constraints (5)-(21) can be solved under alternative specifications:

- Under $\rho^{3}$, for $K_{n}^{f}=K_{n^{-}}{ }^{\Sigma} K^{f 1}{ }_{n}^{+}{ }^{-} X^{i}{ }_{i}{ }_{n^{-}} k_{i}\left(t_{n}-t_{n^{-}}\right)^{\Sigma}$, the stochastic program is linear and solved with CPLEX dual simplex method,

$$
\text { - Under either } \rho^{1} \text { or } \rho^{2} \text {, for } K_{n}^{f}=K_{n^{ \pm}}^{f} K^{f 1}{ }_{n^{+}} \text {> - }{ }_{t} X^{i} X_{j}^{j} k_{i \dot{x}}\left(t_{n_{n}-} t_{n^{-}}\right) \text {, }
$$

we have a convex stochastic program that can be solved as a quadratic program (CPLEX QCP) or adopting a conic solver (e.g. CONOPT) or by switching to CONOPT, taking the quadratic solution as hot start (all solvers are accessible through GAMS 24.6.1).

In the case of a nonlinear stochastic program we benchmark the CPLEX quadratic solver with a combination of CPLEX and the conic solver CONOPT. After extensive testing, due to CONOPT's lack of convergence on very large-scale problems, we have tested it's convergence taking as input the quadratic solution: we present in the following section comparative numerical results and in appendix an extended set of economic evidences, which support however the adoption of CPLEX' quadratically constrained solver without a subsequent switching to the conic solver thereafter.

From a computational viewpoint, any of the above stochastic programs is first implemented in algebraic form using the modeling language GAMS, then the coefficient tree process for the ALM instance is generated internally with Matlab from an imported risk factors' scenario tree based on a corporate economic scenario generator. We use Matlab to derive first the complete set of ALM coefficients and then through a GAMS interface to generate a deterministic equivalent problem Consigli and Dempster (1998). All runs are based on a VMWare Virtual machine with Intel Xeon processor E5620 and 2.40Ghz, 1 physical and 6 virtual processors running on Windows 8Pro x64 operating system with $120 \mathrm{~Gb}$ of hard disk and 8GB of RAM. All problems are solved under the same coefficient and return tree processes.

The solution of the SP problem in Sect. 2 generates a remarkable information output, associated with the tree processes for actuarial and investment variables, their associated ratios, the portfolio revision process and so forth. Here below, however, relying on a specific case-study, we focus on a set of evidences associated with the risk-adjusted returns and risk capital evolutions. The results are generated by a real- 
world case problem with normalized data for confidentiality reasons. We describe in dedicated subsections:

Section 4.2 The case problem dimension and associated generation and solution times in the linear, quadratic and second order conic cases.

Section 4.3 The key statistical properties of the asset classes from which the asset returns tree processes are derived and the regulatory-compliant correlation matrix $\rho^{2}$ adopted in the case-study.

Section 4.4 A comparative study of the main evidences collected in terms of strategic asset and risk capital allocations under different correlation assumptions. Specifically:

Section 4.4.1 The initial optimal portfolio allocation and key evidences on the strategic asset allocation over the planning horizon,

Section 4.4.2 A comparative analysis of the return and risk capital dynamics associated with the three correlation matrices first along a representative scenario, and then

Section 4.4.3 Taking the IRC, the IVS and the RoRAC cumulative distributions at the 5 year horizon into account,

Section 4.4.4 Selected results on the IRC evolution under different correlation assumptions, from which we can infer informations on the processes (25) and (26).

\subsection{Case study}

We consider a 10-year ALM problem with a symmetric 768 scenario tree and branching degree: $\left\{6,4^{2}, 2^{3}\right\}$ The IVS target is set at $1500 \mathbf{e}$ at the end of the third year and the RoRAC target at $20 \%$ at the end of the decision horizon. An initial portfolio worth $100000 \mathbf{e}$ is given and the management has set $\$ 50 \%$ in the objective function. Turnover constraint $\vartheta$ is at $30 \%$ and will limit buyings and sellings since the root node. Furthermore $k^{a} \neq 6 \%$ to determine the TRC in Eq. (20) and $\mathrm{K}=8 \%$ for the cost of capital in the Eq. (13). The corporate tax is at $32 \%$, leading to $\oplus 68 \%$ in Eq. (13). The problem is assumed to be solved on January 2, 2017.

\subsection{SP dimension and solution times}

Table 1 clarifies for given problem formulation, the numerical performance of the adopted solution approaches. After extensive testing and due to extremely slow convergence of CONOPT on large scale problems, we selected CPLEX QCP as preliminary solver then switching to CONOPT.

We show in appendix that the solution of the conic problem is well approximated by the quadratic solution, which however can be reached much faster as reported in Table 1. Accordingly we concentrate on the outputs of the quadratic solver in what follows.

Following the problem specification in (4) under the constraints (5) to (21), we assume a portfolio manager seeking a minimal expected shortfall with respect to the 
Table 1 PC problem dimension and solution times

\begin{tabular}{|c|c|c|c|c|c|}
\hline $\begin{array}{l}\text { SCENARIO TREE } \\
\text { tree structure } \\
\text { Scenario Gen CPU time (s) }\end{array}$ & $\begin{array}{l}768 \\
6-4-4-2-2-2 \\
463\end{array}$ & & & & \\
\hline Correlation model & $\rho_{1}$ & & $\rho_{2}$ & & $\rho_{3}$ \\
\hline Solution algorithm & CPLEX QCP & $\mathrm{QCP}+\mathrm{CONOPT}$ & CPLEX QCP & $\mathrm{QCP}+\mathrm{CONOPT}$ & CPLEX LP \\
\hline \multicolumn{6}{|l|}{ DetEqv MPS file: } \\
\hline Rows & 366,170 & 363,230 & 365,683 & 383,323 & 364,213 \\
\hline Columns & 515,246 & 513,776 & 513,290 & 530,930 & 511,820 \\
\hline Coeff non zero & $4,455,863$ & $4,426,460$ & $4,476,647$ & $4,517,807$ & $4,475,177$ \\
\hline Rows (after presolve) & 91,670 & 87,286 & 90,348 & & 75,015 \\
\hline Columns (after presolve) & 123,015 & 118,927 & 124,277 & & 104,312 \\
\hline Coefficients (after presolve) & $1,255,879$ & $1,201,450$ & $1,278,791$ & & $1,210,776$ \\
\hline Num quadr constraints & 4648 & & 4903 & & na \\
\hline Solution time (CPU time secs) & 702.19 & $19,812.8$ & 399.5 & 5557.5 & 563.3 \\
\hline
\end{tabular}

Table 2 Asset-specific risk and reward statistics for the case study: $\mu_{i}$ are expected returns per annum of assets $i, \sigma_{i}$ the associated standard deviations, $s k_{i}$ their skewness and $k u r_{i}$ their curtosis

\begin{tabular}{lllllllll}
\hline Asset Class & Investment & $\mu_{i}$ & $\sigma_{i}$ & $\frac{\mu_{i}}{\sigma_{i}}$ & $s k_{i}$ & $k u r_{i}$ & $k_{i}$ & $\frac{\mu_{i}}{k_{i}}$ \\
\hline$A_{1}$ & Barcaps & 0.051 & 0.047 & 1.085 & 0.492 & 5.130 & 0.062 & 0.823 \\
& Tips & 0.048 & 0.064 & 0.750 & 0.121 & 3.161 & 0.055 & 0.873 \\
$A_{2}$ & Barclays Inv. grade & 0.055 & 0.037 & 1.486 & 0.379 & 4.934 & 0.064 & 0.859 \\
& Barclays High yield & 0.068 & 0.053 & 1.283 & -0.315 & 6.092 & 0.177 & 0.384 \\
$A_{3}$ & MSCI EMU & 0.090 & 0.206 & 0.437 & 0.272 & 2.837 & 0.390 & 0.231 \\
$A_{4}$ & US Alt Index & 0.115 & 0.081 & 1.419 & 0.439 & 12.790 & 0.490 & 0.235 \\
$A_{5}$ & GPR Europe & 0.175 & 0.243 & 0.720 & -1.333 & 10.159 & 0.250 & 0.700
\end{tabular}

Given the returns per unit volatility $\frac{\mu_{i}}{\sigma_{i}}$, the risk coefficients $k_{i}$ define the tail returns-at-risk from which we derive the risk-adjusted returns $\frac{\mu_{i}}{k_{i}}$. We use weekly data from January 2002 to December 2016

given targets under an extended set of constraints. We focus on a unique scenario set for asset returns and liabilities, and by switching from $\rho^{2}$ to $\rho^{1}$ and $\rho^{3}$ derive comparative results and assess the behavior of $X_{n, \beta}^{o}, K_{n, \rho^{h}}^{f}, \mathrm{v}_{n, \rho^{h}}^{1}$ and $\Psi_{n, \rho^{h}}$.

\subsection{Assets risk-return statistics}

We present in Table 2 a set of descriptive statistics which characterize the assets' returns adopted in the case study. We indicate in the second column the market indices associated with each investment opportunity: Barcaps are the Barclays Treasury indices, Tips the Treasury inflation-linked 5year maturity indices for $A_{1}$, then Barclays Inv.Grade and High-yield indices for $A_{2}$, Morgan Stanley equity (MSCI) for for $A_{3}$, the US Alternative Index for $A_{4}$ and the real estate index GPR Europe for $A_{5}$.

The above statistics must be taken with care, since they correspond to frequency distributions, which neglect any parametric assumption or associated stochastic model. The scenarios adopted in the case study are generated for those asset returns through 
Table 3 Solvency II compliant regulatory correlation matrix

\begin{tabular}{llllllll}
\hline & Barcaps & Tips & Inv grade & High yield & Altern. & Equity & Real Est. \\
\hline Barcaps & 1 & 0.749 & 0.739 & 0.250 & 0.263 & 0.236 & 0.024 \\
Tips & 0.749 & 1 & 0.739 & 0.527 & 0.472 & 0.278 & 0.261 \\
Inv grade & 0.739 & 0.739 & 1 & 0.723 & 0.623 & 0.492 & 0.454 \\
High yield & 0.250 & 0.527 & 0.723 & 1 & 0.921 & 0.822 & 0.765 \\
Altern. & 0.263 & 0.472 & 0.623 & 0.921 & 1 & 0.910 & 0.757 \\
Equity & 0.236 & 0.278 & 0.492 & 0.822 & 0.910 & 1 & 0.716 \\
Real Est. & 0.024 & 0.261 & 0.454 & 0.765 & 0.757 & 0.716 & 1 \\
\hline
\end{tabular}

a corporate scenario generator. Nevertheless the $k_{i}$ coefficients and the correlation matrix in Table 3 do correspond to the data structure actually employed in practice. The expected returns per unit risks in Table 2 help understanding the relevance of fixed income allocations in the optimal portfolios generated by the SP solution, as clarified below. Without loss of generality we may assume that the vector return generating process is stationary over the 10 yearhorizon.

The tail coefficients $k_{i}$ have been estimated by historical simulation from January 2002 until December 2016: they correspond to the historical returns-at-risk at the $0.5 \%$ percentile, $k_{i} E_{i}^{-1}(0.005)$ where $F$ is asset- $i$ returns' marginal probability distribution. A value of $6.2 \%$ for the Treasury bonds implies on that asset class a 1 over 200 potential loss greater than that over 1-year. To derive the $k_{i j}^{h}$ values in Eq. (16) we consider the matrices $\rho_{i j}^{h}$ : all three matrices have only nonnegative correlation coefficients. In general asset returns may very well be negatively correlated but such condition is ruled out by the regulatory framework to enforce a more conservative risk management approach. We consider in Table 3 the adopted regulatory matrix $\rho^{2}$ in the case study.

We show in Sect. 4.4 the collected evidences in terms of IVS and RoRAC performances as the assumption on asset correlations vary.

\subsection{Risk-adjusted performance: a comparative study}

We consider next the evidences on the optimal SAA and associated risk-adjusted return dynamics over the decision horizon for $\rho \rho^{1}\left\{, \rho^{2}, \rho^{3}\right.$. We summarize upfront the key evidences collected in the case-study from a qualitative standpoint:

- Under the different correlation assumptions the optimal policies achieve the requested risk-adjusted targets in most of the scenarios, despite underlying rather different capital requirements.

- The allocated investment risk capital remains relatively low until the 5 year horizon under any specification of $\rho^{h}$.

- The adoption of an SP approach based on the minimisation of the shortfall with respect to the targets is in general very effective in generating relevant capital savings and consistent profits over the 10 year horizon for $h=1$ and $h=2$. 
Table 4 Optimal SAA under different correlation matrices and lower and upper policy bounds

\begin{tabular}{llrrrrrr}
\hline$A_{j}$ & $X_{n 0, \rho}^{o} h$ & $\rho^{1}(\%)$ & $\rho^{2}(\%)$ & $\rho^{3}(\%)$ & $x_{i, n_{0}-}(\%)$ & $l_{i}(\%)$ & $u_{i}(\%)$ \\
\hline \multirow{2}{*}{$A_{1}$} & Cash & 3 & 3 & 3 & 4 & 3 & 8 \\
& Barcaps & 32 & 39 & 33 & 33 & 0 & 100 \\
\multirow{2}{*}{$A_{2}$} & Tips & 1 & 0 & 0 & 3 & 0 & 100 \\
& Inv grade & 23 & 23 & 29 & 23 & 0 & 100 \\
$A_{3}$ & High yield & 24 & 23 & 19 & 25 & 0 & 100 \\
$A_{4}$ & Equity & 3 & 3 & 3 & 3 & 0 & 20 \\
$A_{5}$ & Alternatives & 6 & 2 & 5 & 1 & 0 & 20 \\
\hline
\end{tabular}

- In presence of highly correlated returns $\left(\rho^{3}\right)$ and under the adopted assumptions and scenarios, it may occur that the regulatory capital might not be sufficient to hedge extremely negative market scenarios.

- Over a long horizon the optimal SAA will in the short term rely on portfolio trading profits and interest rate risk immunisation and over the long run increasingly on unrealised portfolio returns and optimal market risk control.

The evidences presented in the following sections are representative of a case-study developed for a PC company and consistent with internal operational requirements.

\subsubsection{Strategic assetallocation}

The implementable here-and-now decision provides, under different correlation assumptions, an optimal strategic asset allocation with respect to the introduced IVS and RoRAC targets.

See Table 4 for the optimal SAA under $\rho^{h}, h=1,2,3$, while $x_{i, n 0}$ defines the asset allocation before the solution of the SP problem.

Solution $X_{n 0, \rho^{2}}^{O}$ specifies the regulatory-compliant optimal root node allocation: relative to the input portfolio there is at the root node a relevant investment on Barcaps funded by selling of Tips and High yield bonds. This decision, we see below, is consistent with a reduction of the asset-liability duration gap and the achievement of the risk-adjusted targets. We interpret the optimal strategic asset allocation at the root node as the reference input to determine a capital allocation consistent with the introduced strategic targets.

Relative to $X_{n 0, \rho^{2}}$, under $\rho^{1}$ or $\rho^{3}$, respectively, we see in the former case a significant increase of investments in Alternatives evenly funded by sellings across the other classes and in the latter case an increased investment in Alternatives and Investment Grade bonds funded by Tips and HY bonds. We show below that the risk-adjusted targets are also reached in these two cases in most of the scenarios and the allocated $\mathrm{RC}$ remains very low. The main evidence here is that as the co-dependence between asset returns changes, so will the strategic decision but with relatively minor effects on the risk capital at least in the short term. 
Table 5 Representativerisk-adjusted return scenarios and investment riskcapital for giveninitial investment of $100 \mathrm{k} \mathbf{e}$

\begin{tabular}{|c|c|c|c|c|c|c|c|}
\hline $\begin{array}{l}\text { Stages } \\
\text { Time horizon (years) }\end{array}$ & $\begin{array}{l}1 \\
0\end{array}$ & $\begin{array}{l}2 \\
0.5\end{array}$ & $\begin{array}{l}3 \\
1\end{array}$ & $\begin{array}{l}4 \\
2\end{array}$ & $\begin{array}{l}5 \\
3\end{array}$ & $\begin{array}{l}6 \\
5\end{array}$ & $\begin{array}{l}7 \\
10\end{array}$ \\
\hline \multicolumn{8}{|l|}{$\operatorname{SQP}\left(\rho^{1}\right)$} \\
\hline$K_{n, \rho^{1}}^{f}(\tilde{\omega})$ & 0 & 1998.19 & 1396.64 & 2900.19 & 3522.28 & 5763.68 & $12,904.48$ \\
\hline$K_{n, \rho^{1}}^{f_{1}}(\tilde{\omega})$ & 0 & 603.13 & 23.38 & 128.23 & 290.83 & 733.06 & 0.00 \\
\hline $\mathrm{v}_{n, \rho 1}^{1}(\tilde{\omega})$ & & -984.52 & -1653.70 & 843.82 & 3617.52 & 7561.28 & 4468.32 \\
\hline$\Psi_{n, \rho^{1}}(\tilde{\omega})$ & & $-0.73 \%$ & $3.78 \%$ & $25.67 \%$ & $33.15 \%$ & $36.97 \%$ & $28.61 \%$ \\
\hline \multicolumn{8}{|l|}{$\operatorname{SQP}\left(\rho^{2}\right)$} \\
\hline$K_{n, \rho 2}^{f}(\tilde{\omega})$ & 0 & 3215.05 & 5464.78 & 3514.51 & 4235.60 & 7936.64 & $19,860.74$ \\
\hline$K_{n, \rho^{2}}^{f_{1}(\tilde{\omega})}$ & 0 & 648.36 & 0.00 & 0.00 & 0.00 & 0.00 & 0.00 \\
\hline $\mathrm{v}_{n, \rho 2}^{1}(\tilde{\omega})$ & & -1339.89 & -518.10 & 924.93 & 4508.54 & $12,059.98$ & 1195.05 \\
\hline$\Psi_{n, \rho^{2}}(\tilde{\omega})$ & & $-3.93 \%$ & $12.40 \%$ & $22.11 \%$ & $30.71 \%$ & $36.45 \%$ & $21.17 \%$ \\
\hline \multicolumn{8}{|l|}{$\operatorname{SLP}\left(\rho^{3}\right)$} \\
\hline$K_{n, \rho 3}^{f}(\tilde{\omega})$ & 0 & 6184.84 & 6017.07 & $13,230.85$ & $13,487.06$ & $23,452.96$ & $82,616.58$ \\
\hline$K_{n, \rho^{3}}^{f_{1}(\tilde{\omega})}$ & 0 & 641.96 & 24.19 & 0.00 & 0.00 & 0.00 & 0.00 \\
\hline $\mathrm{v}_{n, \rho_{3}}^{1}(\tilde{\omega})$ & & -1163.75 & -860.81 & 817.25 & 3318.06 & 6798.15 & $38,921.27$ \\
\hline$\Psi_{n, \rho^{3}}(\tilde{\omega})$ & & $0.34 \%$ & $9.46 \%$ & $17.82 \%$ & $22.23 \%$ & $24.57 \%$ & $35.94 \%$ \\
\hline
\end{tabular}

\subsubsection{A representative scenario optimal policy}

Consider now a representative scenario, denoted by $\tilde{\omega}$ : this is selected after the solution as the scenario from the root to a leaf node leading at the horizon to an average investment profit. Table 5 displays the evolution of the investment risk capital components and risk-adjusted returns along such mean scenario.

According to the evidences in Table 5 every problem is solved with the requested targets achieved under scenario $\tilde{\omega}$.

Consider now in Fig. 1 on the upper plot the evolution of the optimal policy $X_{\rho^{2}}{ }^{o}(\tilde{\omega})$ along scenario $\tilde{\omega}$ and just below the associated IRC evolution. Unlike in Table 5 in the figure we display the cumulative RC estimate. The dotted lines on the lower plot refer to the capital at risk that would have been associated with the two cases of $\rho^{1}$ and $\rho^{3}$ under the same portfolio strategy. The thick red line is the $\hat{\bar{K}}_{\rho^{2}}^{f}(\tilde{\omega})$ maximum allowed risk exposure. Along this scenario we see that since the second stage the investment in high-yield bonds reduces rapidly in favour of Barcaps and Investment grade bonds.

We can summarise the key evidences of this comparative analysis along a single average scenario.

- The optimal portfolio allocation provides an effective hedge of the interest rate risk, for which since the first year a negligible capital protection is needed; 


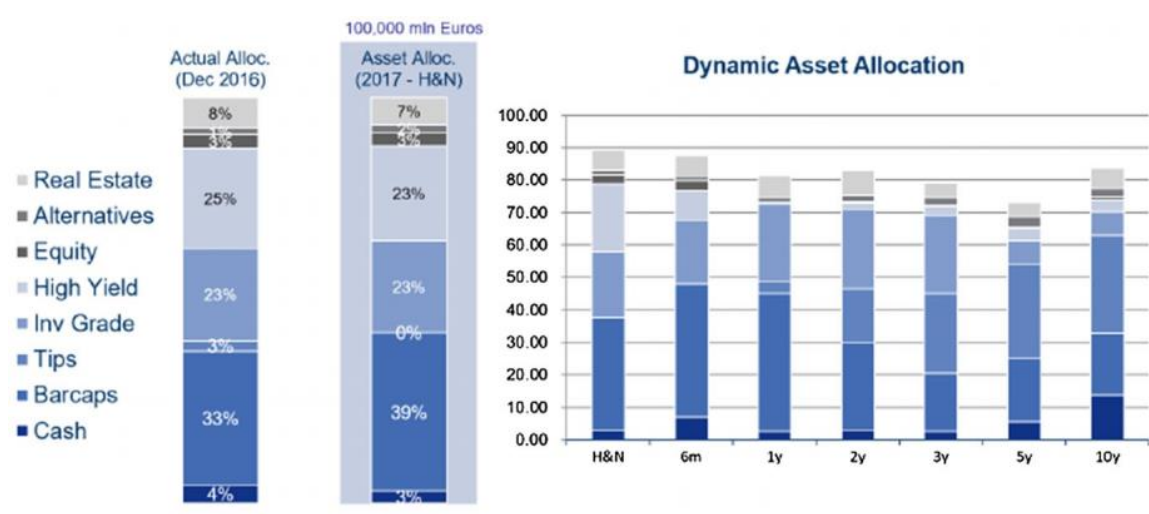

Investment Risk Capital Bounds

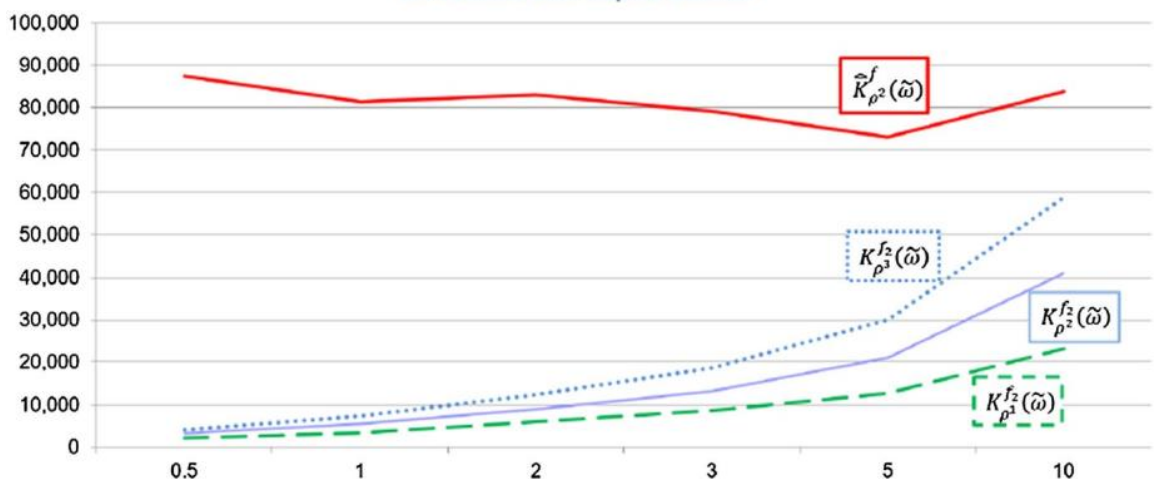

Fig. 1 Top: optimal H\&N policy $X_{n 0, \rho^{2}}^{o}$ (second column, the first refers to the input portfolio $x_{i, n_{0}-}$ ) and mean scenario strategy under correlation assumption $\rho^{2}$. In the lower figure associated risk capital evolution: $K_{\rho^{h}}^{f}(\tilde{\omega}), h=1,2,3$

- Under stressed market conditions, the estimated RC increases significantly but the portfolio strategy tends to compensate such increase with increased profits and the risk-adjusted targets are reached;

- Under any correlation assumption the RC remains very low, witnessing an effective risk diversification over time;

\subsubsection{Risk-adjusted performance and correlations}

The analysis can be extended to evaluate the investment risk capital required at a given risk horizon across all scenarios. The following graphical analysis allows a direct comparison between the IRC distribution at a 5 year horizon and the two risk-adjusted returns distributions for $\rho=\phi^{1}, \rho^{2}, \rho^{3}$. $\}$ This is the information to be considered to assess the capital protection at the beginning of the last stage. We concentrate on this medium term to limit the approximation error induced by the 5-year last stage length. 


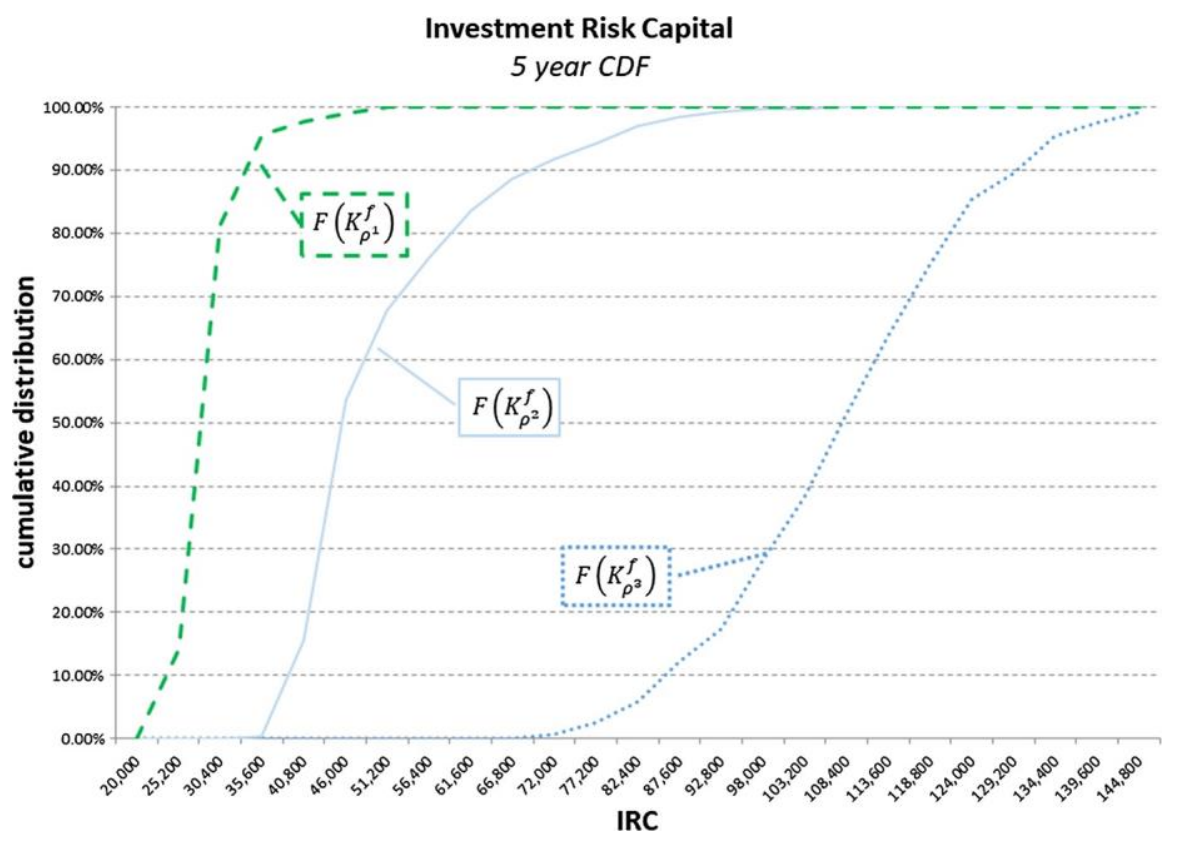

Fig. 2 IRC cumulative distributions for different correlation matrices $\rho^{h}, h=1,2,3$

Figure 2 displays the cumulative distribution functions (cdf) for $K^{f}$ at the beginning of the last stage generated by the optimal solutions under the assumed three correlation matrices.

The cdf's in Fig. 2 are associated with three different optimal solutions and they correspond to the following risk-adjusted return distributions. We show in Fig. 3 the cdf's for the RoRAC again at the 5 year horizon: $F\left(\Psi_{\rho^{1}}\right)$ dominates to the first order $F\left(\Psi_{\rho^{2}}\right)$ and this again to the first order $F\left(\Psi_{\rho^{3}}\right)$. This outcome is attained without imposing any SD constraints in the problem. It is just reported as a statistical evidence worth remarking.

Consider now in Fig. 4 the evidence on the 5-year IVS cumulative distributions.

From above the following relevant remarks can be derived:

- Under any correlation assumption the risk-adjusted targets are achieved when evaluated at the 5 year horizon: the same evidence applies at the 3-and 10-year horizons for the IVS and the RoRAC respectively;

- The IVS CDFs are very close to each other under the three assumptions while the RoRAC CDFs shift to the right when relaxing the correlation assumption: this is due to the accounting within the RoRAC of unrealized portfolio gains in addition to trading profits (common to IVS and RoRAC);

- The RC estimates differ significantly: under $\rho^{1}$ the capital protection requested by the optimal strategy is minimal and increases significantly as we move to $\rho^{2}$ and $\rho^{3}$ : in all cases however from the above two remarks the generated returns compensate the increased capital-at-risk. 


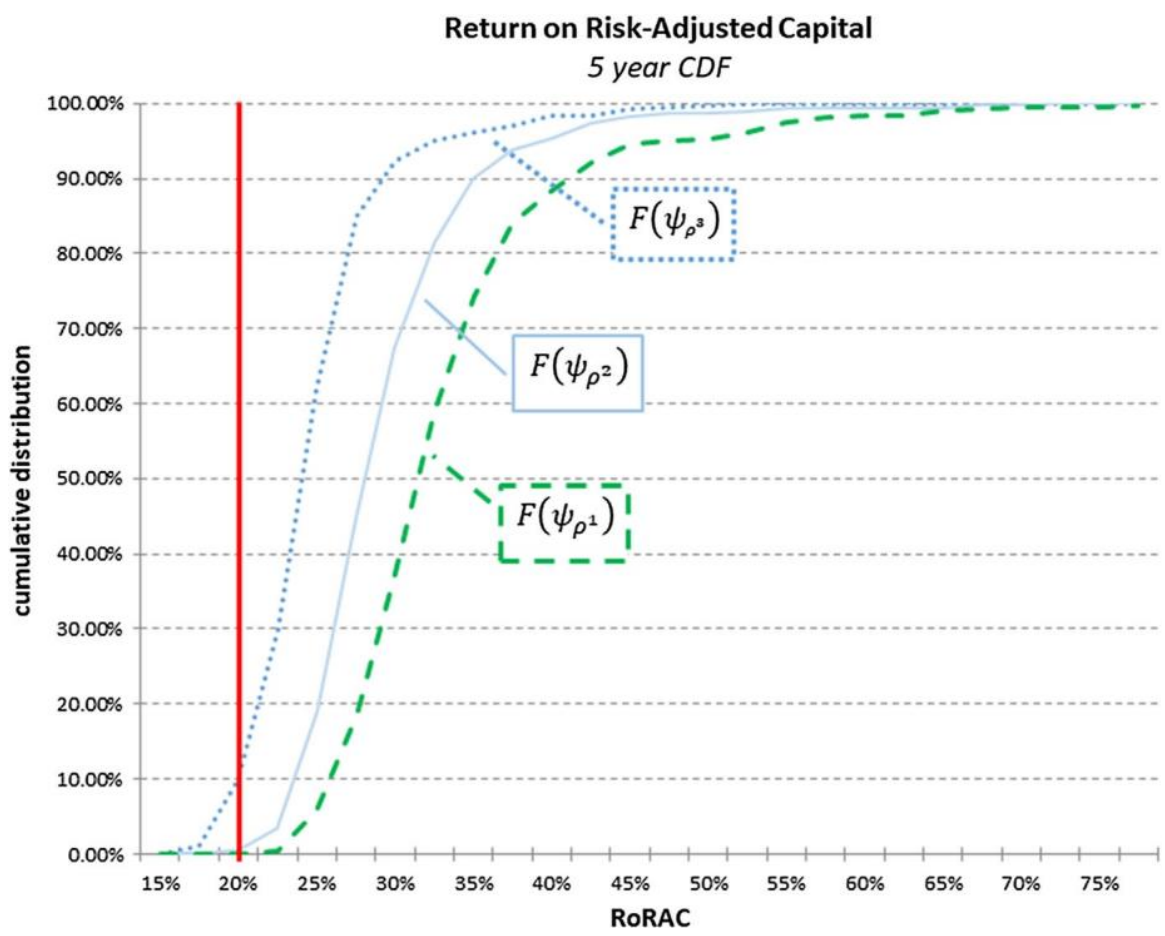

Fig. 3 Return on Risk-adjusted capital cumulative distributions for different correlation matrices $\rho^{h}$, t $1,2,3$ and RoRAC target

- Under any of the above assumptions we have a negligible contribution of interest rate risk to the estimated investment $\mathrm{RC}$.

We complete the analysis on risk-adjusted performances and allocated risk capital by providing in Appendix a set of tables underlying the above cdf's.

\subsubsection{Evidence on risk allocation}

We consider in this final session the evidences collected on the two processes $\delta_{n}^{f}$ in (26) and $\mathrm{Y}_{n}^{f}$ in (25). For given $\rho^{h}$ consider the associated $X_{h}^{o}{ }_{h} \quad$ and $K_{\rho h}^{f}$ and their evolution over the planning horizon across all scenarios. We label with $\omega_{\mathrm{wcs}}$ and $\omega_{b c s}$ those scenarios that according to the IRC evolution lead respectively to the highest and lowest RC estimates at the end of the 10years.

The analysis is relevant to the PC management as well as to regulators. PC managers in particular are interested to minimise risk capital allocation and have a sufficient hedge under any financial scenario. Regulators when validating a risk capital approach must be able to assess the costs induced by their regulations: excessive capital requirements may easily lead to market instability and to negative businesscycles.

We show in Fig. 5 the evolution of the $\delta_{\rho h}^{f}$ processes in Eq. (26) to evaluate the cost of regulatory capital and the one associated with a stressed market condition. Notice 


\section{Investment Value Surplus}

5 year $C D F$

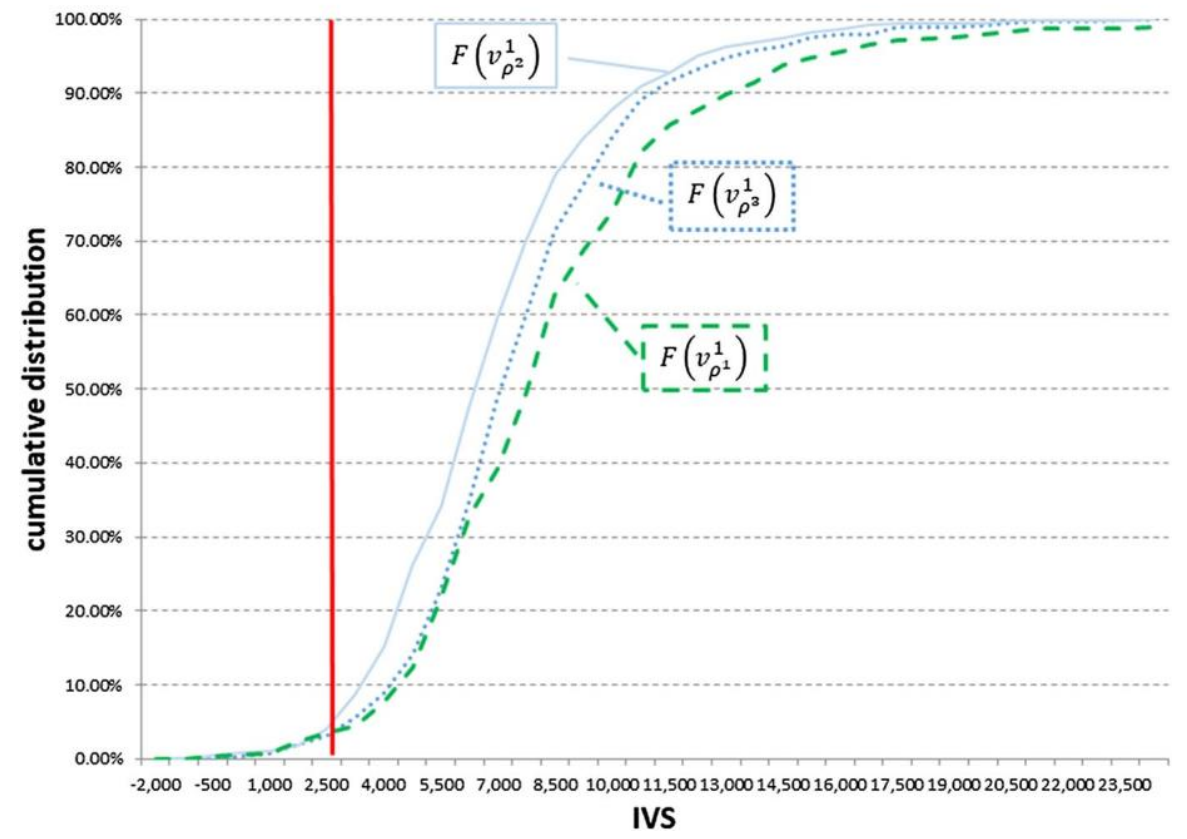

Fig. 4 Investment value surplus cumulative distributions for different correlation matrices $\rho^{h}, \not h 1,2,3$ and IVS target

how effective in terms of minimal capital requirements is the optimal strategy when assuming $\rho_{1}$.

Given a maximum risk exposure $\hat{\bar{K}}_{\rho^{2}}^{f}(\tilde{\omega})$ we see in Fig. 5 that a scenario may occur under which a the end of the 10-year horizon under a conservative correlation assumption $\rho^{3}$ the portfolio may not be worth enough. Up to the 5-year horizon under any correlation assumption an optimal investment strategy would not be expensive in terms of capital protection. From Fig. 5 it is interesting that under $\rho^{2}$ even along a worst-case-scenario the portfolio would be sufficient to hedge any loss with the given confidence interval. Notice that in the short term, 1 to 3 years, the optimal policy would lead to substantial capital savings under any $\rho^{h}$.

Consider now the processes $\mathrm{Y}_{h+1, h}^{f}$, for $h=1,2$ in Fig. 6 . They quantify across all scenarios the differences between internal RC allocations when solving the ALM problem assuming upfront a given $\rho^{h}$ and then comparing pairwise the RC requirements of the associated optimal portfolios $X_{\rho^{h}}^{o}, h=1,2,3$ :

Consider first the thick blue line for $\mathrm{Y}_{2, \mathrm{i}}^{f}$ we see that the maximum $\mathrm{RC}$ increase over the 10 years may reach $60 \%$ of the initial portfolio value and around $15 \%$ over the first 5 years. It is possible that no additional RC may be requested, however, checking the bottom line. When analysing the range of variation of $\mathrm{Y}_{3,2}^{f}$ instead we can see first that the maximum RC incremental cost might be as high as $80 \%$ of the initial portfolio 


\section{Risk capital savings}

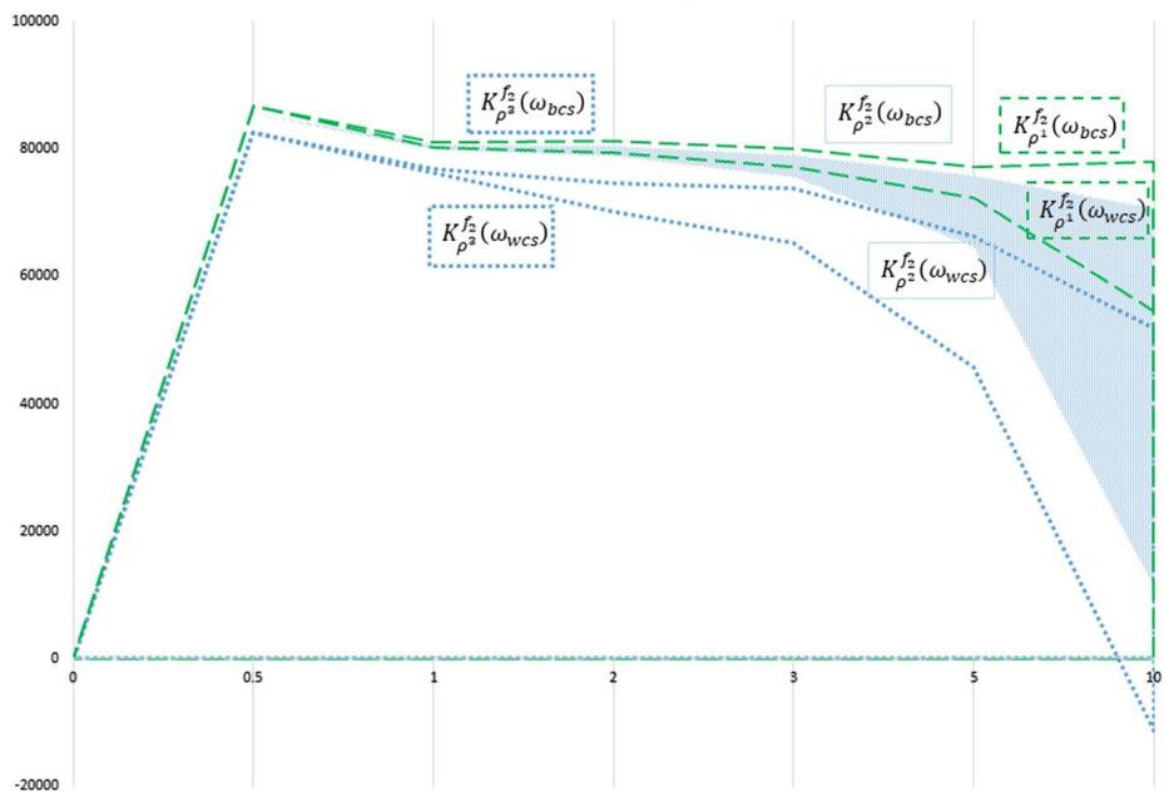

Fig. 5 Risk capital buffer $-\delta_{\rho^{h}}^{f}, h=1,2,3$ in Eq. (26)

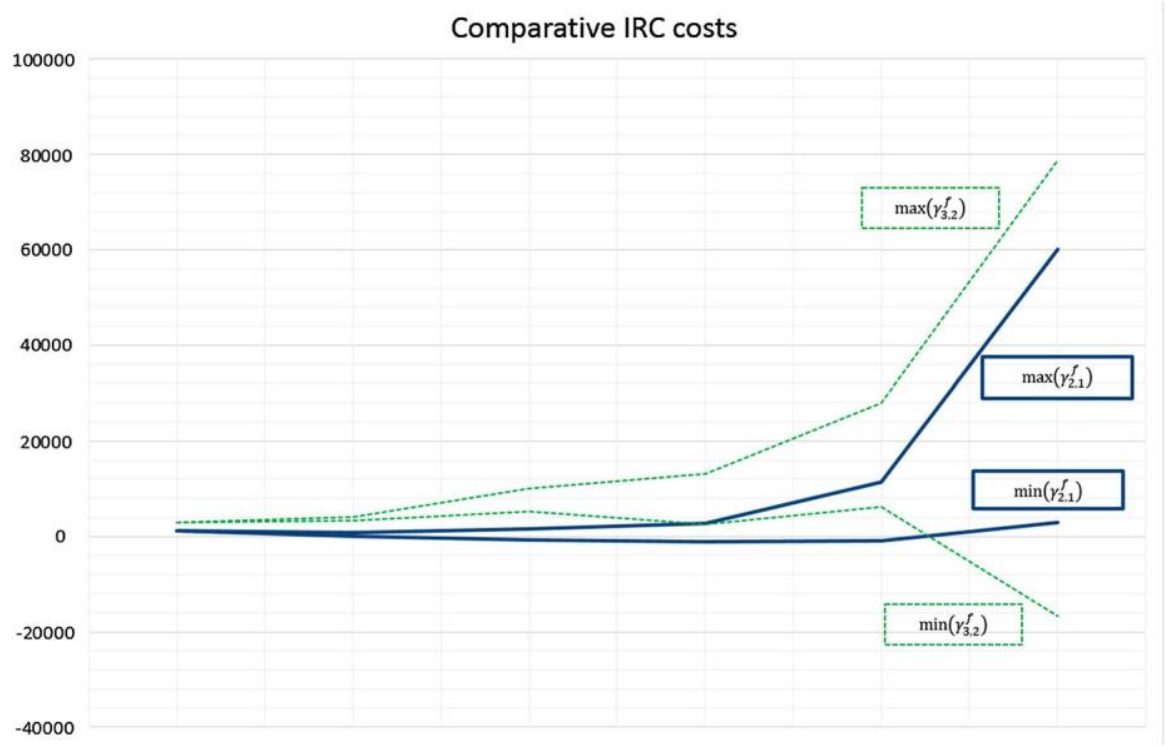

Fig. 6 Optimal strategies incremental IRC $-\gamma_{2,1}^{f}$ and $\gamma_{3,2}^{f}$ in Eq. (25) 
at the end of the planning horizon, while it might be possible that a very conservative investment strategy when assuming $\rho^{3}$ might lead to a net RC decrement relative to a regulatory-compliant strategy.

We can summarize the key evidences from Figs. 5 and 6:

- The investment portfolio can in most cases hedge any large loss up to the end of the planning horizon when $\rho=\rho^{1}, \rho^{2}$ while in presence of perfectly correlated asset returns a loss larger than the upper bound may occur under a particularly negative scenario.

- Over the short and medium term under any scenario and correlation assumption the optimal portfolio strategy requires a limited amount of capital, leading to significant capital savings. The PC company is solvent and the capital cost induced by the regulations is limited.

- Under a very negative market scenario the company may be unable to allocate sufficient capital and should declare default. Such remote event however is estimated at a 10 year horizon and there is sufficient time to protect the company solvability.

- In an optimal RC allocation problem based on a regulatory-compliant correlation matrix, the incremental cost generated by $\rho^{2}$ is limited and consistent with an efficient SAA by the PC manager.

The above evidences support the adoption of an SP formulation based on riskadjusted performance measures and it is of interest to regulators whose stress-testing applications aim at evaluating the companies' distance to default under highly stressed market conditions.

\section{Conclusions}

The original motivation of this article can be found in the widespread adoption of riskbased capital frameworks by financial intermediaries and Institutional investors in most advanced economies (e.g. Basel III agreements in banking sector, Solvency II in the insurance sector and now increasingly in Pension funds and so forth) and the resulting stream of contributions, with wide methodological and modeling implications. Here we have considered a detailed Institutional ALM problem for a global PC insurer and analyzed under relatively standard statistical assumptions the implications of a RC constraint on the optimal ALM strategies.

The transition to the new regulatory system implied on one hand the adoption of a unified modeling approach and on the other led to the introduction of risk-adjusted performance measures in the decision model. The main focus of the article is the ALM model formulation and a thorough analysis of the relationship between return generation and risk exposure under different assumptions on market correlations. The notion of risk capital and the dynamic interaction between optimal strategic asset and capital allocations have been central in the analysis. A computational study on the sensitivity of the optimal solution to three reference correlation hypotheses was presented in the second part of the article.

The following can be regarded as main research goals and contributions to the current state-of-the-art. 
- From a modeling viewpoint: the formulation of a comprehensive multistage Institutional asset-liability management model integrating, beyond the 1-year horizon, the key functions of strategic asset allocation and optimal capital allocation by a global PC portfolio manager. The quantitative evaluation of the investment risk capital components under a standard modeling approach.

- From an insurance economics perspective: the evidence that the inclusion of IVS and RoRAC targets in a multistage recourse model leads to an optimal trade-off between profit generation and risk capital allocation. The inclusion of unrealized gains and losses in the definition of the RoRAC has a positive impact on the long-term profitability of the portfolio. The evaluation relevant to insurance managers and regulators of the costs associated with changing assumptions on assets' correlations.

- From a regulatory perspective, the analysis has clarified the trade-off between increasing capital requirements and PC company solvency.

- The adoption of the optimal root node decision as reference diversification input for capital allocation consistently with the assigned return targets. Such optimal capital distribution criterion may be compared with other practically relevant approaches as in Buch et al. (2011).

- From a computational viewpoint: the computational study was based on the generation of a set of large scale stochastic programs allowing an interesting comparative study between a linear and a quadratic programming formulation. The latter was reported to lead to very similar results to the nonlinear conic solver, but with a significant gain in terms of CPU times.

The development of the case study in Sect. 4.4 focuses on a real-world application with normalized data of a large PC portfolio strategic ALM problem. The relationship between risk-adjusted performance and risk capital evolution was studied under the regulatory constraint of non negative correlations among risk factors, thus bounding from below the potential effects on capital allocation of market based hedging policies.

\section{Appendix A: Comparative risk analysis}

A1: Solution outputs from SQP and SLP instances for $\rho=\rho_{1}, \rho_{2}, \rho_{3}$.

Here next in Tables 6, 7 and 8, we present selected statistics on the cumulative investment profit $\Pi^{f}$, the investment risk capital $K^{f}$, the IVS $\mathbf{v}^{1}$ and the RoRAC $\psi$ tree processes generated by the optimal solutions for $\uparrow\left\{\rho^{1}, \rho^{2}, \rho^{3}\right\}$, respectively from time 0 to $t \neq 0$ years. The reported values clarify the effectiveness of the risk control in each problem instance: under uncorrelated returns portfolio diversification is expected to play a key role in determining high returns per unit risk: the opposite is expected to occur under perfectly positive correlations. Under a linear risk exposure, on the other hand, the portfolio policy is expected to seek a maximum expected return at the cost of high profits volatility. The output analysis that follows is considered as a relevant step in the practical validation of a strategic model for capital allocation and financial planning by institutional investors. 
Table 6 Solution output of 768 scenario problem when $\rho=\rho^{1}$, CPLEX QCP solver: range of values for Cumulative Investment Profit, IRC, IVS and RoRAC

\begin{tabular}{|c|c|c|c|c|c|c|c|}
\hline & & 0.5 & 1 & 2 & 3 & 5 & 10 \\
\hline \multicolumn{8}{|l|}{$\Pi_{\rho^{1}}^{f}$} \\
\hline $\operatorname{Max}$ & 0 & 2343.568 & 6924.871 & $12,421.65$ & $32,154.2$ & $83,803.04$ & $111,785.1$ \\
\hline Mean & 0 & 2037.414 & 4590.441 & 9335.43 & $16,972.53$ & $30,112.49$ & $36,676.46$ \\
\hline Min & 0 & 1740.0121 & 3806.642 & 7528.153 & $11,712.48$ & $19,619.48$ & $14,987.44$ \\
\hline SD & 0 & 219.755 & 648.388 & 1027.102 & 2773.251 & 7698.285 & $15,664.47$ \\
\hline \multicolumn{8}{|l|}{$K_{\rho^{1}}^{f}$} \\
\hline Max & 0 & 1998.188 & 4216.721 & 8193.128 & $12,342.4$ & $19,229.45$ & $50,823.39$ \\
\hline Mean & 0 & 1998.188 & 3792.038 & 6944.382 & $10,142.52$ & $15,488.01$ & $28,558.66$ \\
\hline Min & 0 & 1998.188 & 3297.673 & 5346.357 & 7452.333 & $11,021.03$ & $21,533.22$ \\
\hline SD & 0 & 3.34E-11 & 337.814 & 840.413 & 1269.387 & 1734.288 & 3795.492 \\
\hline \multicolumn{8}{|l|}{$v_{\rho 1}^{1}$} \\
\hline Max & 0 & -55.116 & 1721.867 & 3323.553 & 8125.137 & $30,207.03$ & $50,523.5$ \\
\hline Mean & 0 & -664.741 & -310.893 & 377.934 & 3643.414 & $\begin{array}{c}6810.183 \\
-106218-27\end{array}$ & -1589.66 \\
\hline Min & 0 & -1338.545 & -2808.56 & -2607.52 & \multicolumn{3}{|c|}{$34.20156-1062.18-27,690$} \\
\hline SD & 0 & 524.543 & 1036.154 & 1185.825 & 1258.446 & 3291.434 & $11,106.55$ \\
\hline \multicolumn{8}{|l|}{$\Psi_{\rho^{1}}$} \\
\hline Max & 0 & 0.1778 & 0.362 & 0.395 & 0.536 & 0.843 & 0.569 \\
\hline Mean & 0 & 0.0742 & 0.159 & 0.225 & 0.293 & 0.330 & 0.235 \\
\hline Min & 0 & -0.0337 & -0.03502 & 0.1018 & 0.221 & 0.2176 & 0.106 \\
\hline SD & 0 & 0.0824 & 0.087 & 0.0504 & 0.0501 & 0.0785 & 0.0782 \\
\hline
\end{tabular}

Table 7 Solution output of 768 scenario problem when $\rho=\rho^{2}$, CPLEX QCP, range of values for Cumulative Investment Profit, IRC, IVS and RoRAC

\begin{tabular}{|c|c|c|c|c|c|c|c|}
\hline & & 0.5 & 1 & 2 & 3 & 5 & 10 \\
\hline \multicolumn{8}{|l|}{$\Pi_{\rho^{2}}^{f}$} \\
\hline Max & 0 & 2377.0136 & 5960.612 & $10,370.91$ & $31,985.58$ & $81,638.01$ & $98,083.58$ \\
\hline Mean & 0 & 1854.318 & 4270.99 & 8163.044 & $17,492.36$ & $29,273.95$ & $33,267.83$ \\
\hline Min & 0 & 1357.765 & 2949.984 & 6800.673 & $12,839.54$ & $20,360.56$ & $14,249.45$ \\
\hline $\mathrm{SD}$ & 0 & 335.0959 & 766.721 & 857.537 & 3229.228 & 6646.598 & $14,675.5$ \\
\hline \multicolumn{8}{|l|}{$K_{\rho^{2}}^{f}$} \\
\hline $\operatorname{Max}$ & 0 & 3215.0528 & 5636.909 & 9916.719 & $15,476.82$ & $29,619.87$ & $103,496.3$ \\
\hline Mean & 0 & 3215.0528 & 5348.49 & 8767.094 & $12,640.15$ & $21,005.94$ & $50,499.9$ \\
\hline Min & 0 & 3215.0528 & 5022.18 & 8105.782 & $11,182.55$ & $16,825.63$ & $35,467.92$ \\
\hline $\mathrm{SD}$ & 0 & $5.9156 \mathrm{E}-12$ & 206.3721 & 428.0237 & 983.8784 & 2305.746 & $12,446.25$ \\
\hline \multicolumn{8}{|l|}{$v_{\rho^{2}}^{1}$} \\
\hline Max & 0 & -55.209 & 1724.599 & 3367.46 & 8127.012 & $30,209.29$ & $50,545.97$ \\
\hline
\end{tabular}


Table 7 continued

\begin{tabular}{lllccccc}
\hline & 0.5 & 1 & 2 & 3 & 5 & 10 \\
\hline Mean & 0 & -665.079 & -307.415 & 381.395 & 3649.257 & 6813.943 & -1597.07 \\
Min & 0 & -1339.887 & -2808.47 & -2607.31 & 34.24148 & -1063.93 & $-27,652.3$ \\
SD & 0 & 524.436713 & 1038.344 & 1189.375 & 1262.522 & 3294.123 & $11,088.27$ \\
$\Psi_{\rho}^{2}$ & & & & & & & \\
Max & 0 & 0.1291 & 0.262 & 0.278 & 0.4382 & 0.7006 & 0.464 \\
Mean & 0 & 0.04806 & 0.1277 & 0.189 & 0.274232 & 0.291 & 0.190 \\
Min & 0 & -0.0392 & -0.0439 & 0.1038 & 0.1929 & 0.1935 & 0.0788 \\
SD & 0 & 0.0681 & 0.0685 & 0.0339 & 0.0349 & 0.0576 & 0.0670 \\
\hline
\end{tabular}

Table 8 Solution output of 768 scenario problem when $\rho=\rho^{3}$, CPLEX dual simplex

\begin{tabular}{|c|c|c|c|c|c|c|c|}
\hline & & 0.5 & 1 & 2 & 3 & 5 & 10 \\
\hline \multicolumn{8}{|l|}{$\Pi_{\rho^{3}}^{f}$} \\
\hline $\operatorname{Max}$ & 0 & 2663.252 & 7330.864 & $14,600.74$ & $47,681.99$ & $76,485.93$ & $114,623.5$ \\
\hline Mean & 0 & 2164.875 & 5134.871 & $10,510.48$ & $19,359.62$ & $33,839.53$ & $70,751.84$ \\
\hline Min & 0 & 1868.204 & 3485.444 & 7890.079 & $13,552.77$ & $23,910.51$ & $42,504.28$ \\
\hline SD & 0 & 267.4135 & 1185.223 & 1609.678 & 3849.173 & 6982.319 & $12,452.87$ \\
\hline \multicolumn{8}{|l|}{$K_{\rho^{3}}^{J}$} \\
\hline Max & 0 & 5015.408 & 9936.166 & $21,232.93$ & $34,439.87$ & $63,968.59$ & $148,556.9$ \\
\hline Mean & 0 & 5015.408 & 9602.211 & $18,296.5$ & $27,733.14$ & $47,202.33$ & $107,767.5$ \\
\hline Min & 0 & 5015.408 & 9335.947 & $16,108.2$ & $22,344.06$ & $34,818.61$ & $67,323.45$ \\
\hline SD & 0 & $6.006 \mathrm{E}-11$ & 231.702 & 1350.795 & 2619.778 & 5505.626 & $15,966.52$ \\
\hline \multicolumn{8}{|l|}{$\mathrm{v}_{\rho^{3}}^{1}$} \\
\hline Max & 0 & 67.354 & 2363.614 & 5879.513 & $14,972.07$ & $26,513.5$ & $47,071.91$ \\
\hline Mean & 0 & -537.332 & -88.926 & 908.669 & 3709.746 & 7545.223 & $19,818.65$ \\
\hline Min & 0 & -1172.514 & -2492.07 & -2062.84 & 1991.991 & -146.529 & -7612.86 \\
\hline SD & 0 & 504.975 & 1096.703 & 1306.255 & 1793.246 & 3305.283 & 8474.481 \\
\hline \multicolumn{8}{|l|}{$\Psi_{\rho^{3}}$} \\
\hline Max & 0 & 0.1337 & 0.259 & 0.3005 & 0.471 & 0.5021 & 0.394 \\
\hline Mean & 0 & 0.06889 & 0.1306 & 0.1773 & 0.227 & 0.246 & 0.258 \\
\hline Min & 0 & 0.0015 & 0.0072 & 0.112 & 0.178 & 0.1580 & 0.1663 \\
\hline SD & 0 & 0.0529 & 0.0565 & 0.0319 & 0.0365 & 0.0456 & 0.0432 \\
\hline
\end{tabular}

The likelihood to record negative IVS values is sensitive to market correlations: unlike the RoRAC which is enterprise-wide, the IVS is directly associated with the investment portfolio profitability and reflects the cost of liquidity charged on the investment division by the top management through the cost of funding. It is thus the only 
variable that depends directly on the adopted management policy and associated capital allocation.

In the following linear case the optimal policy is determined by the same return scenarios as in the previous two cases but with a higher cost of capital induced by the unit correlation matrix. Accordingly the investment economic capital is rather expensive but the optimal strategy is able to compensate by generating higher profits and keeping the returns per unit risk consistently high.

We report descriptive statistics from the optimal solution of the SQP-CONOPT solver to the problem $\left(X^{O}, K_{\rho_{1}}, \rho_{1}\right)$ based on an assumption of uncorrelated risk

factors. The table can be compared with Table 5 in the text, where the quadratic solver only was used.

A.2: Here next in Tables 9 and 10 the same statistics from the optimal solutions of the SQP-CONOPT solver to the problem associated with $\rho^{2}$ generated with risk factors evolving according to regulatory based correlation matrix, to be compared with the data in Table 7.

Table 9 Solution output of SQP768 scenario problem when $\rho=\rho^{1}$, CPLEX QCP + CONOPT

\begin{tabular}{|c|c|c|c|c|c|c|c|}
\hline & & 0.5 & 1 & 2 & 3 & 5 & 10 \\
\hline \multicolumn{8}{|l|}{$\mathrm{v}_{\rho}^{1}$} \\
\hline Max & 0 & 282.9602 & 1085.575 & 2731.865 & 3556.813 & $20,091.9$ & $63,237.79$ \\
\hline Mean & 0 & -631.45 & -414.765 & 222.4574 & 1672.886 & 6097.223 & -1431.1 \\
\hline Min & 0 & -1452.65 & -2487.21 & -2220.75 & 1500 & -2954.43 & $-22,015$ \\
\hline SD & 0 & 614.5684 & 960.7772 & 992.9329 & 355.0488 & 3304.681 & $11,015.2$ \\
\hline \multicolumn{8}{|l|}{$\Psi_{\rho^{1}}$} \\
\hline Max & 0 & 0.178027 & 0.227005 & 0.269185 & 0.327401 & 0.531144 & 0.532032 \\
\hline Mean & 0 & 0.048673 & 0.122338 & 0.183404 & 0.225426 & 0.274674 & 0.191467 \\
\hline Min & 0 & -0.06971 & -0.02576 & 0.095996 & 0.183412 & 0.17158 & 0.073317 \\
\hline SD & 0 & 0.088518 & 0.066682 & 0.032372 & 0.027956 & 0.060175 & 0.073452 \\
\hline \multicolumn{8}{|l|}{$K_{\rho^{1}}^{f}$} \\
\hline Max & 0 & 2455.042 & 5648.525 & $12,443.98$ & $19,528.51$ & $34,761.74$ & $79,657.65$ \\
\hline Mean & 0 & 2455.042 & 4692.517 & 9004.504 & $14,131.78$ & $24,682.65$ & $52,768.49$ \\
\hline Min & 0 & 2455.042 & 4099.67 & 7136.29 & $10,121.21$ & $16,190.41$ & $30,441.91$ \\
\hline SD & 0 & $1.96 \mathrm{E}-11$ & 559.7077 & 1622.097 & 2567.862 & 4754.968 & $11,058.09$ \\
\hline \multicolumn{8}{|l|}{$\Pi_{\rho^{1}}^{f}$} \\
\hline Max & 0 & 3839.52 & 6323.131 & $17,259.31$ & $25,552.52$ & $54,214.13$ & $99,900.16$ \\
\hline Mean & 0 & 2123.629 & 4457.811 & 8803.591 & $14,398.39$ & $27,820.74$ & $32,469.14$ \\
\hline Min & 0 & 1514.049 & 3078.819 & 6531.439 & 9215.031 & $15,434.62$ & 8505.77 \\
\hline SD & 0 & 786.9967 & 930.7576 & 1379.246 & 2650.781 & 5987.908 & $15,558.81$ \\
\hline
\end{tabular}


Table 10 Solution output of 768 scenario problem when $\rho=\rho^{2}$, CPLEX QCP-CONOPT

\begin{tabular}{|c|c|c|c|c|c|c|c|}
\hline & & 0.5 & 1 & 2 & 3 & 5 & 10 \\
\hline \multicolumn{8}{|l|}{$v_{\rho^{2}}^{1}$} \\
\hline Max & 0 & 33.69314 & 948.3695 & 3657.493 & 5029.651 & $19,510.52$ & $52,645.41$ \\
\hline Mean & 0 & -525.942 & -197.685 & 554.2437 & 2555.727 & 6687.225 & -2786.39 \\
\hline Min & 0 & -1221.08 & -2541.43 & -2303.64 & 1500 & -660.542 & $-23,837.4$ \\
\hline SD & 0 & 501.8103 & 920.8766 & 1078.685 & 835.6447 & 2878.85 & $12,396.12$ \\
\hline \multicolumn{8}{|l|}{$\psi_{\rho^{2}}$} \\
\hline Max & 0 & 0.129433 & 0.183924 & 0.245687 & 0.288375 & 0.507129 & 0.388739 \\
\hline Mean & 0 & 0.07038 & 0.124596 & 0.171281 & 0.2141 & 0.247784 & 0.166852 \\
\hline Min & 0 & -0.00205 & 0.001258 & 0.092404 & 0.169737 & 0.164695 & 0.076269 \\
\hline $\mathrm{SD}$ & 0 & 0.05183 & 0.046899 & 0.02737 & 0.023495 & 0.044398 & 0.064354 \\
\hline \multicolumn{8}{|l|}{$K_{\rho^{2}}^{f}$} \\
\hline Max & 0 & 5183.92 & 9785.816 & $18,235.28$ & $29,129.4$ & $53,654.05$ & $140,905.9$ \\
\hline Mean & 0 & 5183.92 & 9347.983 & $16,698.76$ & $24,731.95$ & $41,229.94$ & $88,897.26$ \\
\hline Min & 0 & 5183.92 & 9097.589 & $15,330.96$ & $21,490.01$ & $32,689.12$ & $63,870.56$ \\
\hline SD & 0 & $5.01 \mathrm{E}-11$ & 228.3717 & 762.7557 & 1677.866 & 4390.02 & $14,981.54$ \\
\hline \multicolumn{8}{|l|}{$\Pi_{\rho^{2}}^{f}$} \\
\hline $\operatorname{Max}$ & 0 & 3044.781 & 7034.066 & $15,647.7$ & $27,078.74$ & $69,949.42$ & $108,333.1$ \\
\hline Mean & 0 & 2291.986 & 4889.275 & 9544.701 & $16,713.43$ & $30,679.84$ & $35,927.33$ \\
\hline Min & 0 & 1744.382 & 3436.893 & 6439.653 & 9650.334 & $19,364.6$ & $12,435.76$ \\
\hline SD & 0 & 487.4457 & 970.5503 & 1527.203 & 3106.84 & 5947.416 & $17,496.38$ \\
\hline
\end{tabular}

\section{References}

Alessandri P, Drehmann M (2010) An economic capital model integrating credit and interest rate risk in the banking book. J Bank Finance 34(4):730-742

Artzner P, Delbaen F, Eber JM, Heath D (1999) Coherent measures of risk. Math Finance 9(3):203-228

Bertocchi M, Consigli G, Dempster MAH (eds) (2011) Handbook on stochastic optimization methods in finance and energy. Fred Hillier International Series in Operations Research and Management Science. Springer, New York

Buch A, Dorfleitner G (2008) Coherent risk measures, coherent capital allocations and the gradient allocation principle. Insur Math Econ 42(1):235-242

Buch A, Dorfleitner G, Wimmer M (2011) Risk capital allocation for rorac optimization. J Bank Finance 35(11):3001-3009

Cariño DR, Kent T, Myers DH, Stacy C, Sylvanus M, Turner AL, Watanabe K, Ziemba WT (1994) The Russel-Yasuda-Kasai model: an asset-liability model for a japanese insurance company using multistage stochastic programming. Interfaces 24(1):24-49

CEA (2016) Cea statistics no. 45. European Insurance in Figures, pp 1-54. www.insuranceeurope.eu

Consigli G, Dempster MAH (1998) Dynamic stochastic programming for asset-liability management. Ann Oper Res 81:131-162

Consigli G, di Tria M, Gaffo M, Iaquinta G, Moriggia V, Uristani A (2011) Dynamic portfolio management for property and casualty insurance, chap 5. In: Bertocchi M, Consigli G, Dempster M (eds) Handbook on stochastic optimization methods in finance and energy, Fred Hillier International Series in Operations Research and Management Science. Springer, New York, pp 99-124 
Consigli G, Iaquinta G, Moriggia V (2012) Path-dependent scenario trees for multistage stochastic programmes in finance. Quant Finance 12(8):1265-1281

Crouhy M, Galai D, Mark R (2000) A comparative analysis of current credit risk models. J Bank Finance 24:59-117

Das S, Tufano P (1996) Pricing credit-sensitive debt when interest rates, credit ratings and credit spreads are stochastic. J Financ Eng 5:161-198

de Lange PE, Fleten SE, Gaivoronski AA (2004) Modeling financial reinsurance in the casualty insurance business via stochastic programming. J Econ Dyn Control 28(5):991-1012

Dempster MAH, Germano M, Medova EA, Villaverde M (2003) Global asset liability management. Br Actuar J 9(1):137-195

Denault M (2001) Coherent allocation of risk capital. J Risk 4:1-34

Dhaene JM, Goovaerts MJ, Kaas R (2003) Economic capital allocation derived from risk measures. N Am Actuar J 7(2):44-56

Dupačová J, Consigli G, Wallace SW (2000) Scenarios for multistage stochastic programmes. Ann Oper Res 100:25-53

European Parliament (2009) European Parliament, Solvency II Directive. http://eur-lex.europa.eu/ LexUriServ/LexUriServ.do?uri=OJ:L:2009:335:0001:0155:EN:PDF

Gaivoronski AA, Hoyland K, de Lange PE (2001) Statutory regulation of casualty insurance companies: an example from Norway with stochastic programming analysis. In: Uryasev S, Pardalos PM (eds) Stochastic optimization: algorithms and applications, vol 54. Kluwer Academic Publisher, Dordrecht, pp 55-85

Jorion P (1996) Value at risk: a new benchmark for measuring derivatives risk. Irwin Professional Pub, Burr Ridge

Maume-Deschamps V, Rullière D, Said K (2015) A risk management approach to capital allocation. arXiv preprint arXiv:1506.04125

Modigliani F, Modigliani L (1997) Risk-adjusted performance. J Portf Manag 23(2):45-54

Mulvey JM, Erkan HG (2003) Simulation for risk management: risk management of a P/C insurance company scenario generation, simulation and optimization. In: Proceedings of the 35 th conference on Winter simulation: driving innovation, pp 364-371

Mulvey JM, Erkan HG (2005) Decentralized risk management for global property and casualty insurance companies. In: Wallace S, Ziemba W (eds) Applications of stochastic programming. SIAM, Philadelphia, pp 503-530

Mulvey JM, Pauling B, Britt S, Morin F (2007) Dynamic financial analysis for multinational insurance companies. In: Zenios S, Ziemba W (eds) Handbook of asset and liability management: applications and case studies, vol 2. North Holland, Amsterdam, pp 543-589

NAIC P/C RBC working group and others (1992) Property-casualty risk-based capital requirement a conceptual framework

Pflug GC, Roemisch W (2007) Modeling, measuring and managing risk, vol 20. World Scientific, Singapore

Pflug GC, Ruszczynski A (2005) Measuring risk for income streams. Comput Optim Appl 32:161-178

Saita F (2010) Value at risk and bank capital management: risk adjusted performances, capital management and capital allocation decision making. Academic Press, Boca Raton

Stoughton NM, Zechner J (2007) Optimal capital allocation using raroc and eva. J Financ Intermed 16(3):312-342

Tasche D (2007) Capital allocation to business units and sub-portfolios: the Euler principle. arXiv preprint arXiv:0708.2542

Weindorfer B (2012) A practical guide to the use of the chain ladder method in non-life insurance. Research Papers of the Bfi Working Paper 77, University of Applie Sc bfi Vienna, pp 1-22 